\title{
Portfolio theory for squared returns correlated across time
}

\author{
Ernst Eberlein · Dilip B. Madan
}

Received: 12 January 2016 / Accepted: 10 June 2016 / Published online: 16 August 2016 (C) The Author(s). 2016 Open Access This article is distributed under the terms of the Creative Commons Attribution 4.0 International License (http://creativecommons.org/licenses/by/4.0/), which permits unrestricted use, distribution, and reproduction in any medium, provided you give appropriate credit to the original author(s) and the source, provide a link to the Creative Commons license, and indicate if changes were made.

\begin{abstract}
Allowing for correlated squared returns across two consecutive periods, portfolio theory for two periods is developed. This correlation makes it necessary to work with non-Gaussian models. The two-period conic portfolio problem is formulated and implemented. This development leads to a mean ask price frontier, where the latter employs concave distortions. The modeling permits access to skewness via randomized drifts. Optimal portfolios maximize a conservative market value seen as a bid price for the portfolio. On the mean ask price frontier we observe a tradeoff between the deterministic and random drifts and the volatility costs of increasing the deterministic drift. From a historical perspective, we also implement a mean-variance analysis. The resulting mean-variance frontier is three-dimensional expressing the minimal variance as a function of the targeted levels for the deterministic and random drift.
\end{abstract}

\section{Introduction}

A persistent empirical observation about equity market returns is that even though they tend to be uncorrelated across two successive time periods, they are not independent, as the squared returns are highly correlated (see for example Barndorff-Nielsen and Shepard (2002) and the references cited therein) with correlation coefficients as high as seventy percent. A natural question, arising as a consequence of such intertemporal correlations, are the portfolio theoretic implications of such facts. In particular, we ask how allocations move between time periods when correlations rise

\footnotetext{
E. Eberlein $(\bowtie)$

University of Freiburg, Freiburg im Breisgau, Germany

e-mail: eberlein@stochastik.uni-freiburg.de

D.B. Madan

University of Maryland, College Park, USA
} 
and do the results also dependent on other underlying market conditions. To answer such questions we consider a three date, two-period model for investment opportunities permitting correlation in squared returns between the periods. More dynamic models including continuous-time models could also be developed (see for example Basak and Chabakauri (2010)), but the simpler two-period model serves as a useful building block. In this regard, we build upon the single-period fundamental portfolio theory of Markowitz (1952, 1991).

For a two-period model it is natural to take the investment criterion as applying to the aggregate two-period return. Since much has been written on the portfolio theory for a single investment period from a mean-variance perspective, we include an analysis of the two-period problem from a similar perspective. A number of authors have considered a multi-period mean-variance formulation (see for example, among others, Acharya and Pedersen (2005), Ait-Sahalia and Brandt (2001), Bansal, et al. (2004), Brandt (2009) Campbell and Viceira (2002), Hong, et al. (2006), Jagannathan and Ma (2003)), but from the myopic perspective of optimizing the one-step ahead objective function. However, we also recognize some of the shortcomings of meanvariance theory with its use of rewards being measured in units that do not tally with those used for risk. Furthermore, the criterion is not arbitrage consistent as a positive cash flow accessed at zero cost is desirable whatever its variance. We are thus led to primarily consider alternative criteria. To limit the analysis we select a single alternative.

The possibilities include expected utility theory, other risk measures like semivariance, value at risk, or the expected shortfall. Expected utility theory does not focus attention on the allocation problem as it seeks to determine the actual size of positions. It is also difficult to accommodate losses with a dimensionless risk aversion coefficient. Some of the other risk measures may be related to special cases of the recently proposed conic portfolio theory objectives proposed in Madan (2016). The criterion of conic portfolio theory is to maximize a conservative future market value viewed as the infimum of valuations taken with respect to a number of candidate test probabilities. The result can be written as the mean less the supremum over a set of probabilities of the expectation for the negative of the centered return. The latter may be seen as a positive market ask price and serves as the embedded risk measure. Being positive, it is bounded below by zero and leads to a natural mean ask price efficiency frontier. The conservative value maximizing portfolio is then located on this frontier at a point where the slope of the frontier is unity. This is because the conservative value is just the difference between the mean and the ask price so the rate of exchange between the mean and the ask price is always unity. Greater risk aversion is accommodated by expanding the set of probabilities with respect to which one takes the infimum in defining the conservative value or, equivalently, the supremum in defining the ask price. We present an analysis of the two-period portfolio problem from the perspective of conic portfolio theory applied to the aggregate two-period return.

Apart from the criterion, one must specify the decision variables and the information set employed in the decision making. In keeping with portfolio theory for a single period, the decision variables for the first period are the dollar investments in the available vector of asset returns for the first period. For the second period, we take the same, supposing that all returns are available for two consecutive periods. 
We build as information for the investment decision the joint law for the returns over two periods, where the law is known and has been estimated at the start of the first period. In particular, there isn't a Markovian structure with the distribution of secondperiod returns responding to actual realizations in the first period. The realizations are seen as quite noisy with little or no impact on the prior estimated distribution for the second period. We recognize that if there was a firmly asserted joint law, then one could be Bayesian and attempt to infer the conditional second-period law to be used for the second-period decision. Such a procedure places considerable faith in the asserted two-period law and our criterion is going to take the infimum over many test probabilities as none of them are believed to be relevant with enough confidence to be used in a Bayesian construction. We therefore commit to second-period investments at the start of the first period and then evaluate the aggregate portfolio return at the end of two periods.

There is an extensive literature applying such precommitment investment policies. We may cite as examples Bajeux-Besnainou and Portait (1998), Bielecki et al. (2005), Cvitanic et al. (2008), Cvitanic and Zapatero (2004), MacLean et al. (2011) and Cochrane (2014). Such precommitment solutions are also obtained in Duffie and Richardson (1991) in a continuous-time incomplete markets setting while Leippold et al. (2004) work in a discrete-time complete markets setting, whereas, Zhou and Li (2000), and Lim and Zhou (2002) approach the problem in a continuous-time complete markets setting. Basak and Chakabauri (2010) are critical of such precommitment policies arguing that they are not likely to be time consistent and leave incentives open for investors to deviate from the precommitment positions. They argue, in line with Strotz (1956), that rational decision making should be time consistent involving plans that will in fact be followed. However, this is time consistency within a model of information evolution that rational decision makers recognize, will be called into question. The incentive to deviate then remains for time consistent solutions as and when the information evolution models are questioned, reformulated or re-estimated. Such super-rationality is not possible for long and at best we may hope to precommit for a short period. The reasons for deviation are many and we focus attention here on just a two-period precommitment.

We recognize that, classically, portfolio problems are analysed in the context of the presence and absence of a risk-free asset. We therefore develop both approaches over two periods from both a conic and mean-variance perspective. However, if the holding period is not locked in, then fixed income securities are exposed to the risk of movements in interest rates and their return is no longer risk free. Many fixedincome investments are spread over multiple maturities with exposure to interest rate risk making them no longer risk-free and then also correlated with equity returns. The more reasonable and economically relevant perspective is then that for the absence of a risk-free asset. In fact, on recognizing that a credit default swap trades on the debt of the US Treasury leads one to conclude that the US Treasury cannot credibly promise a future dollar. In which case no one can. A risk-free asset is then a fiction that perhaps has outlived its usefulness.

Many solutions to optimal portfolio problems involve numerical methods as opposed to exact closed forms (see for example Ait-Sahalia and Brandt (2001), Brandt et al. (2005), Brandt and Santa Clara (2006) among others). The conic 
portfolio optimization problem is numerically tractable. We also reduce the twoperiod mean-variance problem to a fixed point problem that is numerically solved.

The outline of the rest of the paper is as follows. Section "Two-period return modeling" takes up the two-period conic portfolio theory and the associated mean ask price frontier. Section "Two-period portfolio theory from a conic perspective" also presents the details for the construction of investment opportunity frontiers for conic portfolio theory allowing access to both correlated squared returns across time periods and access to random drifts that leverage the design of skewness in portfolio construction. Section "Conservative value maximizing portfolios across two-periods with correlated squared returns" takes up the implementation of two-period conic portfolio theory. Section "Two-period mean-variance analysis" presents an analysis of the problem from the mean-variance perspective developing the two-period portfolio theory along with the two-period mean-variance frontier and the procedures for its computation. Section "Conclusion" concludes. All proofs are provided in the Appendix.

\section{Two-period return modeling}

Consider the context of an initial wealth $V_{0}$ invested in $n$ risky assets. Let the firstand second-period risky asset returns be given by random $n$-dimensional vectors of $R_{1}, R_{2}$, respectively. If the time zero and time one dollar investments in the $n$ assets are given by $n$-dimensional vectors $a_{0}, a_{1}$, respectively, then the random wealth accumulated at the end of the second period is

$$
V_{2}=V_{0}\left(1+a_{0}^{\prime} R_{1}\right)\left(1+a_{1}^{\prime} R_{2}\right) .
$$

With a view towards accomodating correlation in squared returns we model returns on the basis of time changed Lévy processes where we correlate the subordinators which are used for the time changes. We therefore write

$$
\begin{aligned}
& R_{1}=X_{1}\left(T_{1}\right)+\mu_{1} \\
& R_{2}=X_{2}\left(T_{2}\right)+\mu_{2},
\end{aligned}
$$

where $X_{1}(t), X_{2}(t)$ are zero-mean multivariate Lévy processes which are assumed to be independent of each other and independent of the time changes $T_{1}$ and $T_{2} . \Sigma_{1}, \Sigma_{2}$ denote the covariance matrices of $X_{1}(1), X_{2}(1)$. In case these variables are onedimensional, we shall write $\sigma_{1}^{2}, \sigma_{2}^{2}$ for their variances. To accomodate correlation in squared returns we model $T_{1}, T_{2}$ as

$$
\begin{aligned}
& T_{1}=G\left(\alpha_{1}\right)+G_{1}\left(1-\alpha_{1}\right) \\
& T_{2}=G\left(\alpha_{2}\right)+G_{2}\left(1-\alpha_{2}\right),
\end{aligned}
$$

for subordinators $G, G_{1}, G_{2}$ and $0 \leq \alpha_{1}, \alpha_{2} \leq 1$. These three processes are assumed to be independent and also independent of $X_{1}, X_{2}$. For reasons of tractability of the model, we shall in the following choose $G, G_{1}$, and $G_{2}$ as gamma processes 
with unit mean rate and variance rate $v, v_{1}, v_{2}$, respectively. To be more precise, the characteristic function of $G(1)$ is

$$
E[\exp (i u G(1))]=\left(\frac{1}{1-i u v}\right)^{\frac{1}{v}}
$$

In the special case of $v_{1}=v_{2}=v$, it is easy to see that the random times $T_{1}, T_{2}$ are themselves gamma distributed with unit mean and variance $v$.

When the processes $X_{i}(t)$ being time changed are Brownian motion with drift $\widetilde{\theta}$, the resulting asset returns have a variance gamma distribution. Let us look at this particular specification from an empirical point of view. For this purpose, introduce two random variables $Z_{1}, Z_{2}$ as standard normal variables where to begin with we admit a nonzero correlation $\rho$.

In the univariate case, centered returns are then modeled as

$$
\begin{aligned}
X_{1} & =\tilde{\theta}\left(T_{1}-1\right)+\sigma \sqrt{T_{1}} Z_{1} \\
X_{2} & =\tilde{\theta}\left(T_{2}-1\right)+\sigma \sqrt{T_{2}} Z_{2} \\
E\left(Z_{1} Z_{2}\right) & =\rho
\end{aligned}
$$

with $T_{1}, T_{2}$ as in Eqs. (3) and (4) for $\nu_{1}=\nu_{2}=v$ and $\alpha_{1}=\alpha_{2}=\alpha$. One may estimate $\sigma, v, \widetilde{\theta}$ from the data on the time series of daily returns. The dependency parameters $\alpha, \rho$ may then be estimated using the $E M$ algorithm by integrating out the hidden variates $G, G_{1}$ and $G_{2}$. We estimated these dependency parameters on 96 stocks of the $S \& P 100$ index and present in Figs. 1 and 2 the estimated values for $\alpha$ and $\rho$, respectively.

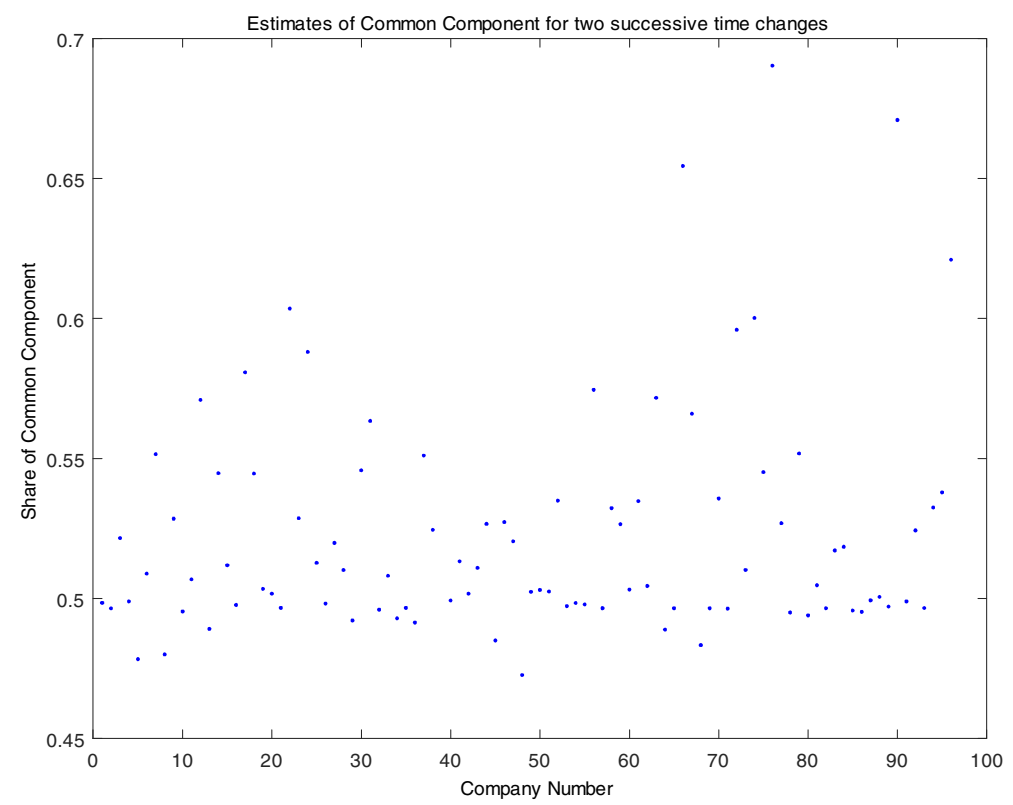

Fig. 1 Estimates of $\alpha$, the share of the common component of the time change 


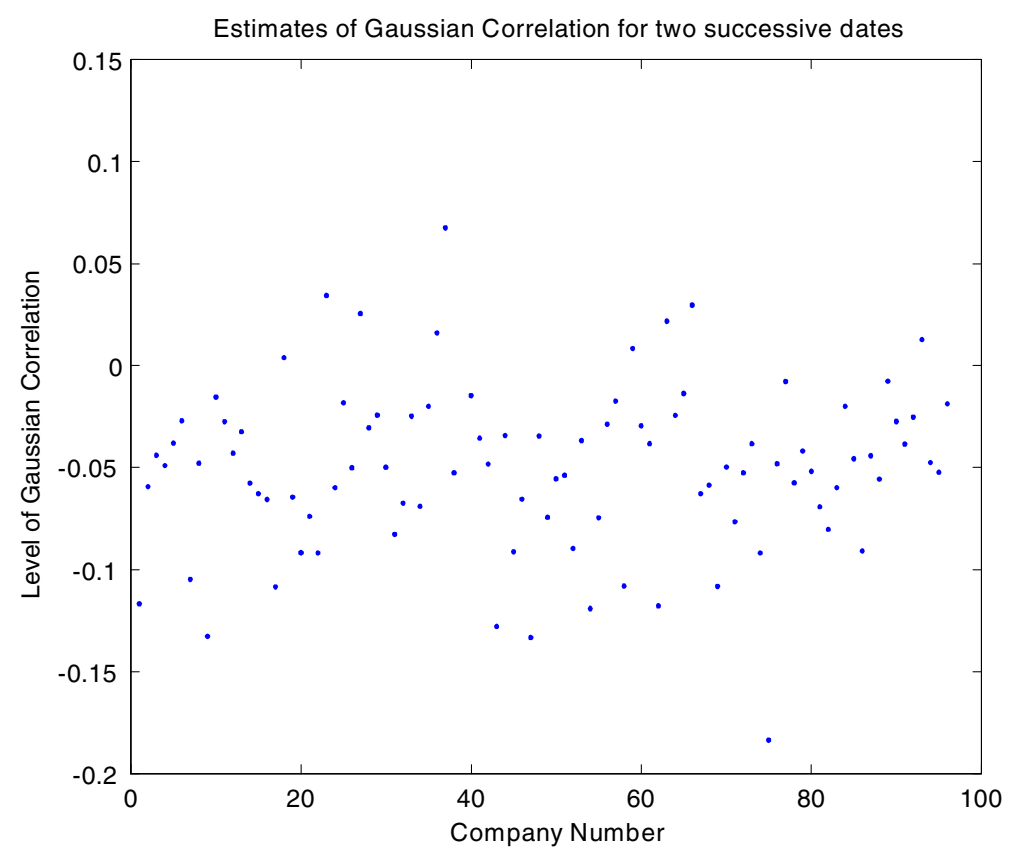

Fig. 2 Estimates of correlation in the Gaussian component

We observe that the Gaussian correlation is low, but the time changes have a significant common component reflecting the correlation expected in squared returns. This leads us to work under the hypothesis that $X_{1}, X_{2}$ are independent of each other, with covariance matrices $\Sigma_{1}, \Sigma_{2}$ for periods one and two, respectively.

\section{Two-period portfolio theory from a conic perspective}

Mean-variance theory is ideally suited to contexts where return distributions are defined by these moments and such a context is provided by multivariate normal return distributions. Under such a hypothesis across two periods the absence of autocorrelation renders returns between periods to be independent. The presence of autocorrelation in squared returns is then inconsistent with this implied independence. Much of the evidence, along with the considerations of correlation in squared returns, points towards non-Gaussian models for returns. What objective functions are then best suited to the task of designing portfolios? Much depends on the purpose of the portfolio design.

With a strict one- or two-period view of the situation, one can imagine the case of an investor placing monies in the market at the start of the period involved, liquidating the position at the end of the period and consuming the resulting accumulated wealth. Axioms of rational behaviour under uncertainty then suggest the use of the expected utility of final wealth as the appropriate decision criterion. However, in 
many practical situations such a formulation misconstrues the reality of the investment activity. The periods involved are fairly short with durations of a few weeks or months at the end of which no consumption of accumulated wealth is being contemplated. Instead, the portfolio is to be liquidated and reinvested into a new portfolio designed in the light of new circumstances and information. Hence, the focus is just on the market value of the portfolio at the future date, marked by the end of the period, and not on its utility, expected or otherwise. Potential market participants could then be primarily interested in maximizing the market value of the portfolio. Now, classically, the market value of a portfolio is the sum of the value of its components and is, as a consequence, independent of how it is constructed. This linearity or additivity follows from the law of one price and the absence of arbitrage opportunities.

Conic portfolio theory is based on the pricing operators of two-price economies where the law of one price is abandoned. Such economies separate bid and ask prices. Markets are viewed as offering to buy random cash flows at prices that render the resulting cash flows less its price to be market acceptable. Acceptable cash flows include all nonnegative cash flows but more generally form a convex cone of acceptable random variables. Every such cone may equivalently be represented as those cash flows that have a positive expectation under a set of test probability measures. As a consequence, the best bid price becomes the smallest expectation delivered by the test probabilities. The bid price being an infimum of expectations is then a concave function and the bid price for a package may well exceed the sum of the bid prices for the components. In conic portfolio theory portfolios are designed to maximize this bid price seen as a conservative market valuation.

Markets are also viewed as willing to sell random cash flows if the resulting price less the cash flow is market acceptable. Positivity of expectations under test probabilities renders the best ask price to be the supremum of expectations across test probabilities. The ask price is then a convex function on the space of random cash flows and, by construction, the ask exceeds the bid. It is also the case that the ask price is the negative of the bid for the negative cash flow.

Since constants come out of the infima of expectations, Madan (2016) shows that one may write the bid price as the expected value under a base probability less the ask price for the negative of the centered or demeaned cash flow. One may then also view the bid price as measuring reward by the expectation under the base probability less a risk measure given by the ask price for the negative of the centered cash flow. Since the centered negative cash flow has a zero mean by construction, and as the base probability is one of the test probabilities, the ask price is always positive and can be minimized subject to attaining a particular expectation. This naturally leads us to a mean ask price frontier and examples of such constructions may be found in Madan (2016). Here we consider the mean ask price frontier for the two-period portfolio problem.

Two additional assumptions termed comonotone additivity and law invariance simplify the evaluation of the bid price of a random cash flow. We suppose the random variables being considered are defined on a probability space $(\Omega, \mathcal{F}, P)$ for a base probability $P$. In order to explain comonotone additivity, we first note that two random variables $X, Y$ are said to be comonotone if, for example, one is a monotone increasing function of the other. More generally they move together in the same 
direction across the set of events, or have no negative comovements or a Kendall's tau of unity. In general, the bid price of $X+Y$ is larger than the sum of the bid prices for each, reflecting some possible advantages of diversification. Comonotone additivity asserts that for comonotone risks we have strict additivity with the bid for the sum equalling the sum of the bids in this case. Put another way, there are no diversification benefits for comonotone risks.

The second assumption of law invariance asserts that the bid price be computable from information on just the probability law of the random cash flow. How the random variable correlates with other random variables is not relevant. This is a strong assumption from the perspective of the concerns of particular agents who may well be interested in whether the cash flow being valued provides hedging benefits for other risks they are already carrying. However, the valuation attained in an abstract market, like that induced by the Walrasian auctioneer who is merely concerned with trying to clear as much risk as possible, may make correlation issues less relevant.

Under these two assumptions Kusuoka (2001) showed that the bid price $b(X)$ of a random variable $X$ with distribution function $F_{X}(x)$ is given by the expectation under concave distortion. More specifically, there exists a concave distribution function $\Psi(u)$ defined on the unit interval such that

$$
b(X)=\int_{-\infty}^{\infty} x d \Psi\left(F_{X}(x)\right) .
$$

The set of test probabilities under which $X-b(X)$ is market acceptable or has a nonnegative expectation are shown in Madan et al. (2015) to be given by all probabilities $Q$ such that for all $A \in \mathcal{F}$

$$
Q(A) \leq \Psi(P(A)) .
$$

Cherny and Madan (2009) observed that expectation under concave distortion is also an expectation under the quantile based change of measure $\Psi^{\prime}\left(F_{X}(x)\right)$. Further requiring that $\Psi^{\prime}(u)$ tends to infinity and zero as $u$ tends to zero or unity, respectively, to reflect both risk aversion and an absence of gain enticement, they introduced the distortion termed minmaxvar and defined, for a stress level parameter $\gamma$, by

$$
\Psi^{\gamma}(u)=1-\left(1-u^{\frac{1}{1+\gamma}}\right)^{1+\gamma} .
$$

We shall use this distortion to illustrate bid price evaluations in this paper.

It is shown below that we may also write

$$
\begin{aligned}
b(X) & =E[X]-a(\tilde{X}) \\
\widetilde{X} & =E[X]-X
\end{aligned}
$$

and the functional $a(X)$ is the ask price functional defined as

$$
a(X)=\int_{-\infty}^{\infty} x d \Psi\left(1-F_{X}(x)\right) .
$$

We may now construct the mean ask price frontier and maximize the bid price on this frontier as the maximum for the mean less the ask price on the frontier. 
For our two-period return in the absence of a risk-free asset we note that

$$
R_{0,2}^{p}=\left(1+a_{0}^{\prime} R_{1}\right)\left(1+a_{1}^{\prime} R_{2}\right),
$$

where $R_{1}, R_{2}$ are as in Eqs. (1) and (2). Furthermore, we have the constraints

$$
\begin{aligned}
& a_{0}^{\prime} \mathbf{1}=1 \\
& a_{1}^{\prime} \mathbf{1}=1 .
\end{aligned}
$$

We may write

$$
R_{0,2}^{p}=E\left[R_{0,2}^{p}\right]+\widetilde{R}_{0,2}^{p}
$$

where $\widetilde{R}_{0,2}^{p}$ is the centered two-period return.

For the bid price we then have

$$
\begin{aligned}
b\left(R_{0,2}^{p}\right) & =E\left[R_{0,2}^{p}\right]+b\left(\widetilde{R}_{0,2}^{p}\right) \\
& =E\left[R_{0,2}^{p}\right]-\left(-b\left(-\left(-\widetilde{R}_{0,2}^{p}\right)\right)\right) \\
& =E\left[R_{0,2}^{p}\right]-a\left(-\widetilde{R}_{0,2}^{p}\right) .
\end{aligned}
$$

We may write

$$
\begin{aligned}
R_{0,2}^{p}= & \left(1+a_{0}^{\prime}\left(R_{1}-\mu_{1}+\mu_{1}\right)\right)\left(1+a_{1}^{\prime}\left(R_{2}-\mu_{2}+\mu_{2}\right)\right) \\
= & \left(1+a_{0}^{\prime} \mu_{1}+a_{0}^{\prime} \widetilde{R}_{1}\right)\left(1+a_{1}^{\prime} \mu_{2}+a_{1}^{\prime} \widetilde{R}_{2}\right) \\
= & 1+a_{0}^{\prime} \mu_{1}+a_{1}^{\prime} \mu_{2}+a_{0}^{\prime} \mu_{1} a_{1}^{\prime} \mu_{2}+\left(1+a_{0}^{\prime} \mu_{1}\right) a_{1}^{\prime} \widetilde{R}_{2}+\left(1+a_{1}^{\prime} \mu_{2}\right) a_{0}^{\prime} \widetilde{R}_{1} \\
& +a_{0}^{\prime} \widetilde{R}_{1} a_{1}^{\prime} \widetilde{R}_{2} .
\end{aligned}
$$

We see that

$$
\widetilde{R}_{0,2}^{p}=\left(1+a_{0}^{\prime} \mu_{1}\right) a_{1}^{\prime} \widetilde{R}_{2}+\left(1+a_{1}^{\prime} \mu_{2}\right) a_{0}^{\prime} \widetilde{R}_{1}+a_{0}^{\prime} \widetilde{R}_{1} a_{1}^{\prime} \widetilde{R}_{2}
$$

and the mean ask price frontier requires the minimization of

$$
a\left(-\widetilde{R}_{0,2}^{p}\right)
$$

subject to

$$
\begin{aligned}
a_{0}^{\prime} \mathbf{1} & =1 \\
a_{1}^{\prime} \mathbf{1} & =1 \\
a_{0}^{\prime} \mu_{1}+a_{1}^{\prime} \mu_{2}+a_{0}^{\prime} \mu_{1} a_{1}^{\prime} \mu_{2} & =m,
\end{aligned}
$$

where $m$ is the target two-period mean return. The bid price maximizing portfolio is the one on this frontier that maximizes the value of $m-a\left(-\widetilde{R}_{0,2}^{p}\right)$.

The bid price could be maximized directly for the optimal portfolio. Alternatively one may also construct the mean ask price frontier analogous to a mean-variance frontier with the advantage that the optimal portfolio is located on the frontier where its slope is unity, as risk and reward are both measured in dollars and the bid price is precisely the reward less the risk of the ask price and there is no trade-off coefficient between the two. The situation with mean-variance is both artificial and arbitrary 
as reward is measured in dollars and risk in squared dollars and the use of a linear trade-off between them quite inappropriate and unsatisfactory.

The solution of this maximization problem requires the specification of the joint law across many assets, say $n$, for the vector of returns simultaneously across two consecutive periods with the resulting distributional problem being one in dimension $2 n$, the dimension of the joint vector $\left(a_{0}, a_{1}\right)$. This is quite a tall order and, with a view to gaining some tractability on this problem, we build our way up to this problem by first reporting on the simpler one-period subproblem. The two-period mean ask price frontier is taken up in the next section. Here we compare the one-period problem in our context with the classical mean-variance frontier that must be revised to accomodate time change conditional drifts differentiated from unconditional drifts.

This subproblem in our context is richer than the classical mean-variance problem by providing access to skewness via the drift of the time changed Brownian motion along with kurtosis via the volatility of the time change. The mean ask price frontier that we eventually employ takes account of all these dimensions of the problem. Even if we fix the kurtosis and consider just the minimization of variance, there are now two drifts to be addressed in the portfolio design for even a single period. They are unconditional mean and the mean conditional on the time change that we call a random drift. As a consequence, we observe that the classical mean-variance frontier for a single-period is now three-dimensional.

For $n$ assets over a single period let the drifts of the Brownian motions to be time changed be given by a vector $\theta$. The centered returns are then modeled by

$$
\widetilde{R}_{1}=\theta(T-1)+\sqrt{T} Z,
$$

where $Z$ is multivariate Gaussian with mean zero and covariance matrix $\Sigma$. The time change represents a measure of economic time and is uniform across assets. Given the law of the time change, to be specified later, we may access the return distribution of any portfolio with centered return

$$
\begin{aligned}
\widetilde{R}_{p} & =a^{\prime} \widetilde{R}_{1} \\
& =d(T-1)+\sqrt{T v} z \\
d & =a^{\prime} \theta \\
v & =a^{\prime} \Sigma a
\end{aligned}
$$

and $z$ is a standard normal variate. In addition, if the assets have mean returns, $\mu$, the portfolio return may be accessed with the knowledge of three numbers $m=a^{\prime} \mu$, the random drift $d$ and the variance $v$ along with the law of $T$. We recognize that these values are related via the equations for $m, d, v$ in terms of the portfolio weights $a$.

To further describe the classical minimum variance investment opportunity set for the first period, we consider the problem

$$
\begin{aligned}
v^{*} & =\min _{a} a^{\prime} \Sigma a \\
\text { s.t. } a^{\prime} \mu & =m \\
a^{\prime} \theta & =d \\
a^{\prime} \mathbf{1} & =1 .
\end{aligned}
$$


The solution to this problem (5) may be described in terms of three distinguished portfolios

$$
\begin{aligned}
& \eta=\frac{\Sigma^{-1} \mu}{\mathbf{1}^{\prime} \Sigma^{-1} \mu} \\
& \delta=\frac{\Sigma^{-1} \theta}{\mathbf{1}^{\prime} \Sigma^{-1} \theta} \\
& \zeta=\frac{\Sigma^{-1} \mathbf{1}}{\mathbf{1}^{\prime} \Sigma^{-1} \mathbf{1}}
\end{aligned}
$$

their mean returns $\rho_{\eta}, \rho_{\delta}, \rho_{\zeta}$; their random drifts $y_{\eta}, y_{\delta}, y_{\zeta}$; and their variances and covariances $\sigma_{\eta}^{2}, \sigma_{\delta}^{2}, \sigma_{\zeta}^{2}, \sigma_{\eta \delta}, \sigma_{\eta \zeta}$ and $\sigma_{\delta \zeta}$.

The solution here may be contrasted with classical mean-variance theory as presented, for example, in Skiadas (2009) Chapter 2 where only the first and the third portfolios are involved in describing the one-dimensional frontier. In the current context, three portfolios are distinguished and the frontier is two-dimensional.

Proposition 1 The solution to problem (5) is given by

$$
v^{*}=\tilde{\lambda}^{2} \sigma_{\eta}^{2}+\widetilde{\kappa}^{2} \sigma_{\delta}^{2}+\tilde{\pi}^{2} \sigma_{\zeta}^{2}+2 \tilde{\lambda} \widetilde{\kappa} \sigma_{\eta \delta}+2 \tilde{\lambda} \tilde{\pi} \sigma_{\eta \zeta}+2 \widetilde{\kappa} \tilde{\pi} \sigma_{\delta \zeta},
$$

where

$$
\begin{aligned}
\tilde{\lambda}\left(\rho_{\eta}-\rho_{\zeta}\right)+\widetilde{\kappa}\left(\rho_{\delta}-\rho_{\zeta}\right)+\rho_{\zeta} & =m \\
\tilde{\lambda}\left(y_{\eta}-y_{\zeta}\right)+\widetilde{\kappa}\left(y_{\delta}-y_{\zeta}\right)+y_{\zeta} & =d \\
1-\tilde{\lambda}-\widetilde{\kappa} & =\tilde{\pi} .
\end{aligned}
$$

We suppose the asset space is rich enough to permit the availability of variances above the minimum variance given $m, d$ with all levels of $m, d$ being attainable. The investment opportunity set for the first period then consists of triples $m, d, v$ with

$$
v \geq v^{*}(m, d)
$$

This is a three-dimensional mean-variance frontier as the optimal variance now depends on the choice of both a deterministic drift $m$ and a random drift $d$.

By way of an example we take the inputs

$$
\begin{array}{ll}
\rho_{\eta} & 0.06 \\
\rho_{\delta} & 0.09 \\
\rho_{\zeta} & 0.03 \\
y_{\eta} & -0.1 \\
y_{\delta} & -0.12 \\
y_{\zeta} & -0.05 \\
\sigma_{\eta} & 0.15 \\
\sigma_{\delta} & 0.20 \\
\sigma_{\zeta} & 0.05 \\
\sigma_{\eta \delta} & 0.0210 \\
\sigma_{\eta \zeta} & 0.0015 \\
\sigma_{\delta \zeta} & 0.0001 .
\end{array}
$$


Figure 3 presents a graph of such a three-dimensional volatility efficiency frontier while Fig. 4 presents an associated contour plot.

This opportunity set is fully defined by specifying the mean returns, random drifts, variances, and covariances of the three distinguished portfolios. The investment opportunity sets may be allowed to be different in the two periods by taking different settings for the mean returns, random drifts, variances, and covariances of the distinguished portfolios in the two periods.

In the presence of risk-free assets for both periods with interest rates of $r_{1}, r_{2}$, respectively, the volatility cost frontier is defined in terms of two distinguished portfolios

$$
\begin{aligned}
\xi & =\frac{\Sigma^{-1}(\mu-r \mathbf{1})}{\mathbf{1}^{\prime} \Sigma^{-1}(\mu-r \mathbf{1})} \\
\delta & =\frac{\Sigma^{-1} \theta}{\mathbf{1}^{\prime} \Sigma^{-1} \theta} .
\end{aligned}
$$

For the frontier one needs the excess returns $x_{\xi}=\xi^{\prime}(\mu-r \mathbf{1}), x_{\delta}=\delta^{\prime}(\mu-r \mathbf{1})$; the random drift coefficients $y_{\xi}=\xi^{\prime} \theta, y_{\delta}=\delta^{\prime} \theta$; the variances $\sigma_{\xi}^{2}, \sigma_{\delta}^{2}$; and the covariance $\sigma_{\xi \delta}$.

Proposition 2 In the presence of a risk-free asset, the minimum variance $v^{*}$ for a deterministic drift of $m$ and a random drift of $d$ is given by

$$
v^{*}=\tilde{\lambda}^{2} \sigma_{\xi}^{2}+\widetilde{\kappa}^{2} \sigma_{\delta}^{2}+2 \tilde{\lambda} \widetilde{\kappa} \sigma_{\xi \delta}
$$

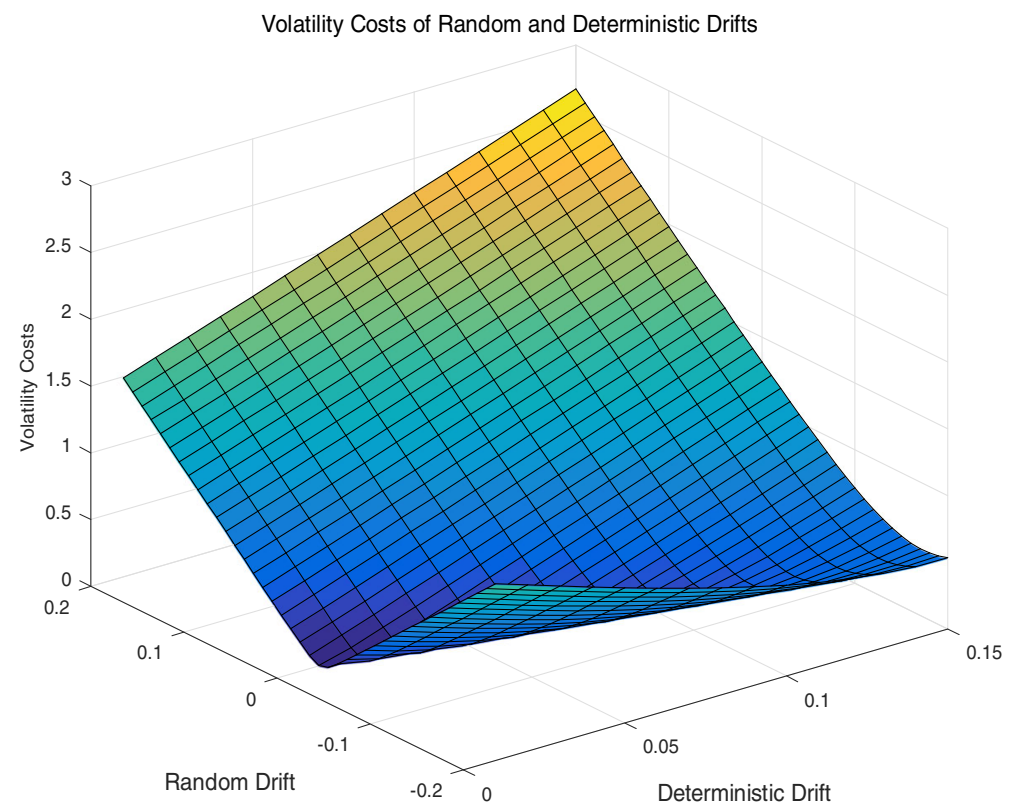

Fig. 3 Volatility costs as a function of the level of random and deterministic drifts 


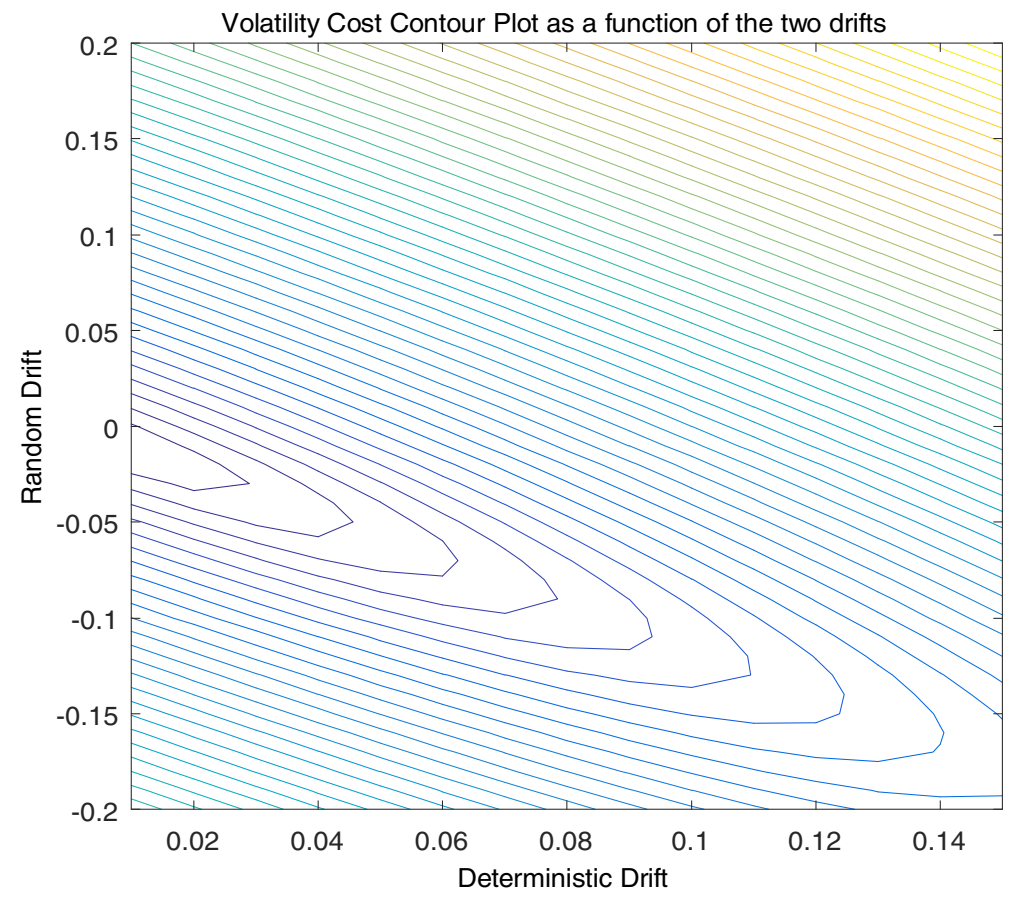

Fig. 4 Contour plot of volatility cost frontier as a function of the two drifts

where

$$
\left[\begin{array}{ll}
x_{\xi} & x_{\delta} \\
y_{\xi} & y_{\delta}
\end{array}\right]\left[\begin{array}{l}
\tilde{\lambda} \\
\tilde{\kappa}
\end{array}\right]=\left[\begin{array}{l}
m \\
d
\end{array}\right]
$$

The ask price minimization problem may be implemented by specifying the joint law for the two time changes in the two periods. In this regard, we first observe that stochastic volatility models as formulated by GARCH models are not able to deliver the level of correlation in squared volatilities observed in data. These correlations can be higher than 0.75 .

In a typical GARCH( 1,1$)$ specification, the squared returns are given by

$$
\begin{aligned}
Y_{1} & =\sigma_{1}^{2} Z_{1}^{2} \\
Y_{2} & =\sigma_{2}^{2} Z_{2}^{2} \\
\sigma_{2}^{2} & =\omega+\beta \sigma_{1}^{2}+\alpha Y_{1} .
\end{aligned}
$$

We may compute the correlation between $Y_{1}, Y_{2}$ for $Z_{1}, Z_{2}$ being independent standard normal variates.

Proposition 3 The correlation between $Y_{1}, Y_{2}$ is bounded above by

$$
\frac{1}{\sqrt{\frac{\beta^{2}}{\alpha^{2}}+4+2 \beta / \alpha}} .
$$


For typical values of $\beta$ near unity and $\alpha$ near zero or $(1-\beta)$ this correlation is expected to be small.

We recognise from Proposition 3 that for a given first-period volatility the only source of correlation is the randomness in first-period squared returns that typically receives a small weight in estimated models. To give the first-period volatility some volatility of its own we simulate the first-period volatility from a log-normal distribution with its own volatility and then compute squared return correlations. Figure 5 presents a graph of squared return correlations as a function of the volatility of the first-period return volatility.

We observe that typical levels of empirically observed squared return correlations are associated with very high levels of first-period log-normal volatility. With deterministic first-period volatility there is no chance for correlations in squared returns to reach empirically observed levels.

These considerations have motivated our use of correlated time changes as the source for correlation in squared returns. Volatility in such a construction is then a random variable going forward and not a number. This introduces the possibility of substantially correlating squared returns.

Figure 6 presents results on simulated squared return correlations for different levels of $v, \alpha$. The returns are generated as

$$
\begin{aligned}
& X_{1}=-0.2\left(T_{1}-1\right)+0.25 \sqrt{T_{1}} Z_{1} \\
& X_{2}=-0.3\left(T_{2}-1\right)+0.32 \sqrt{T_{2}} Z_{2}
\end{aligned}
$$

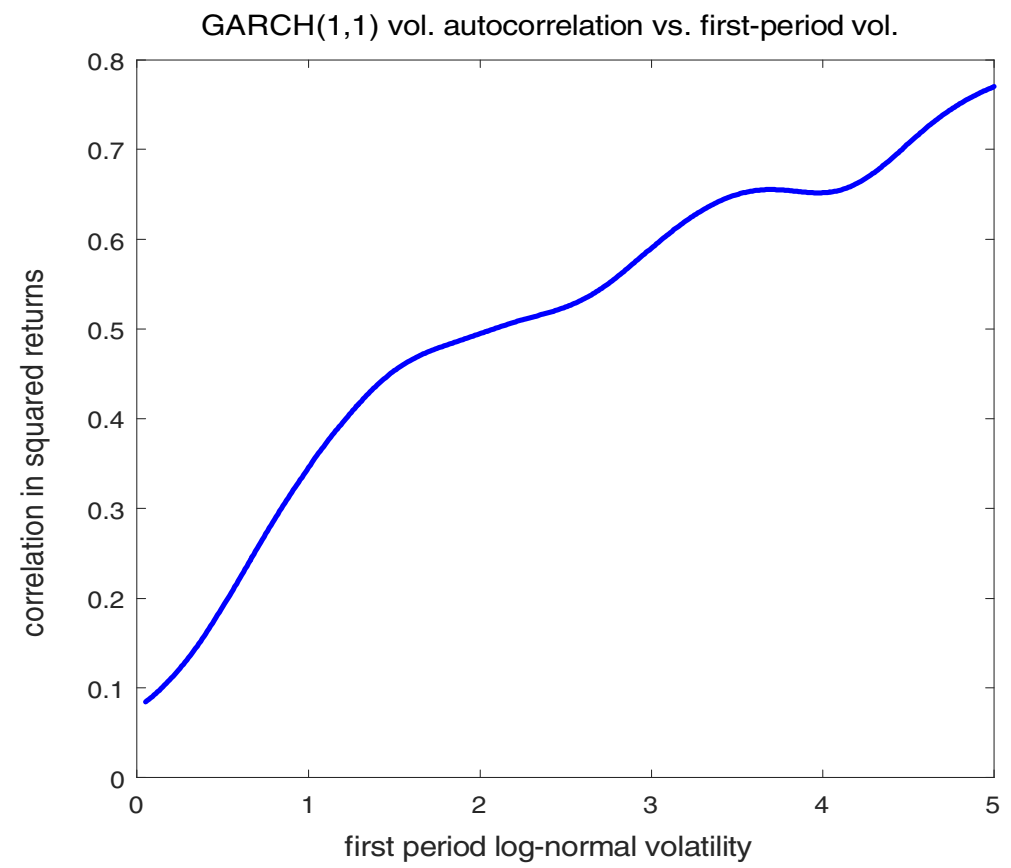

Fig. 5 Correlation in squared returns as a function of the log-normal volatility of first-period volatility 


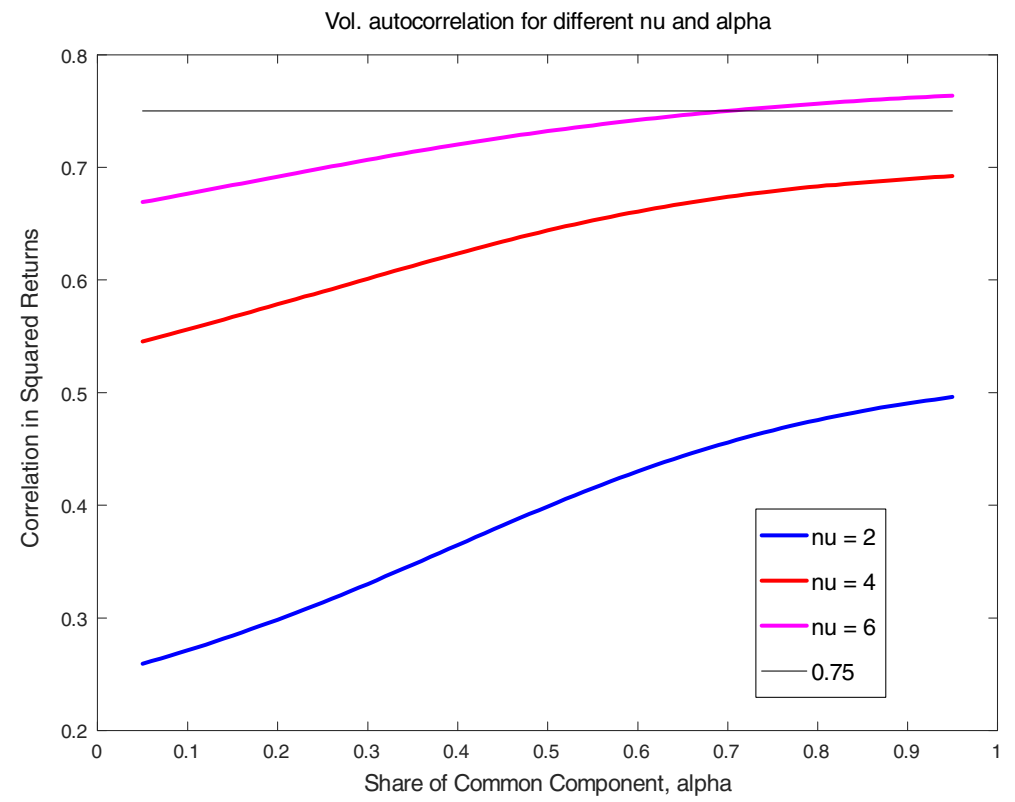

Fig. 6 Squared return correlations for a sample of time changes

$$
\begin{aligned}
& T_{1}=G(\alpha)+G_{1}(1-\alpha) \\
& T_{2}=G(\alpha)+G_{2}(1-\alpha) .
\end{aligned}
$$

$G(t), G_{1}(t), G_{2}(t)$ are independent gamma processes with mean rates unity and variance rates $v, 1,1, Z_{1}$ and $Z_{2}$ are standard normal variates independent of each other and $G, G_{1}, G_{2}$. Though we deal with processes we are only interested in the associated random variables.

With such a formulation for the correlated time changes in the two periods one may solve for the mean ask price frontier by minimizing the ask price for a given level of the two-period mean return. In the process we determine the deterministic drifts $m_{1}, m_{2}$; the random drifts $d_{1}, d_{2}$; and the portfolio variances $v_{1}, v_{2}$. The conservative value maximizing or bid price maximizing portfolios may then be located on this frontier. The next section implements this program.

\section{Conservative value maximizing portfolios across two-periods with correlated squared returns}

As before we take the time changes to be drawn from gamma processes. We set the proportion of the common time change to 0.25 with a variance rate of the common time change at 3 while the independent components have a variance rate of unity. This choice was motivated by Fig. 6 where such a setting pushes the correlation in squared returns near to 0.5 even though the share of the common component is below 
what was estimated in data. The two frontiers with no risk-free asset were defined by the setting used for Fig. 3 for the first period. The setting for the second period was given by the following parameter choices

$$
\begin{array}{ll}
\rho_{\eta} & 0.07 \\
\rho_{\delta} & 0.10 \\
\rho_{\zeta} & 0.02 \\
y_{\eta} & -0.15 \\
y_{\delta} & -0.10 \\
y_{\zeta} & -0.07 \\
\sigma_{\eta} & 0.18 \\
\sigma_{\delta} & 0.30 \\
\sigma_{\zeta} & 0.07 \\
\sigma_{\eta \delta} & 0.0324 \\
\sigma_{\eta \zeta} & 0.0039 \\
\sigma_{\delta \zeta} & 0.0042 .
\end{array}
$$

For a stress level of $\gamma=0.275$ we construct the two-period mean ask price frontier. The frontier along with the value maximizing portfolio and its details are presented in Fig. 7. The six variables for $m_{1}, m_{2}, d_{1}, d_{2}, v_{1}, v_{2}$ were found by a nonlinear constrained optimization with the variances constrained to lie above the minimum values corresponding to the choices for $m, d$ in each period as is consistent with the frontier for the period. The Figures report the standard deviations or the square roots of the variances.

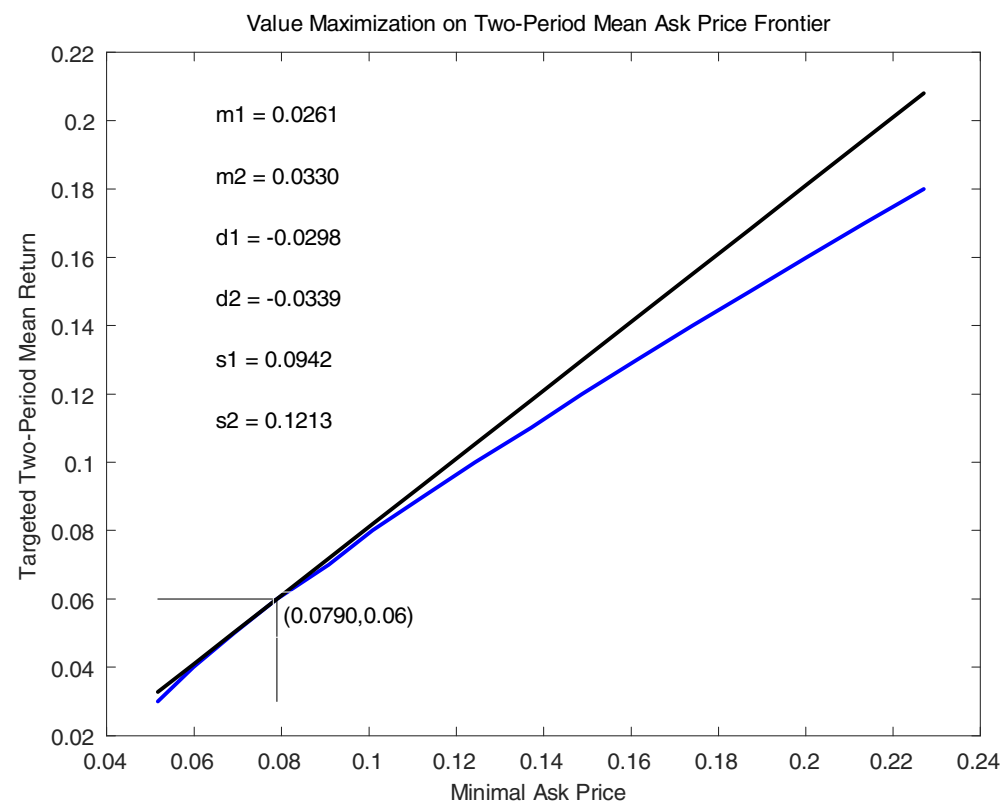

Fig. 7 Value maximizing frontier and value maximizing portfolio with no risk-free asset 
We observe in this case that the second-period deterministic drifts are higher as are the volatilities. The random drifts targeted are negative with a greater skewness in the second period. Figure 8 presents the solution for the same frontiers but for an increased share of common component of 0.3 .

We observe that the targeted mean rate for the two periods is unchanged, but the first period has a higher mean return and the second a lower one. The negative skewness and volatilities also rise in the first period and fall in the second.

Additionally, we present in Figs. 9 and 10 the mean returns targeted in each period against the random drift and the volatility, respectively, for both periods. We observe a tradeoff between the deterministic and random drift and the volatility cost incurred for higher mean returns.

In the absence of access to skewness or when $\theta$ equals zero for each period, the mean ask price frontier in the absence of a riskless asset is more curved. Figure 11 presents an example of such a frontier.

\section{Two-period mean-variance analysis}

For a mean-variance analysis of two-period returns we develop the equations for the two-period mean return and its variance.

Proposition 4 The two-period mean return is given by

$$
E\left[\frac{V_{2}}{V_{0}}\right]=1+a_{0}^{\prime} \mu_{1}+a_{1}^{\prime} \mu_{2}+a_{0}^{\prime} \mu_{1} a_{1}^{\prime} \mu_{2} .
$$

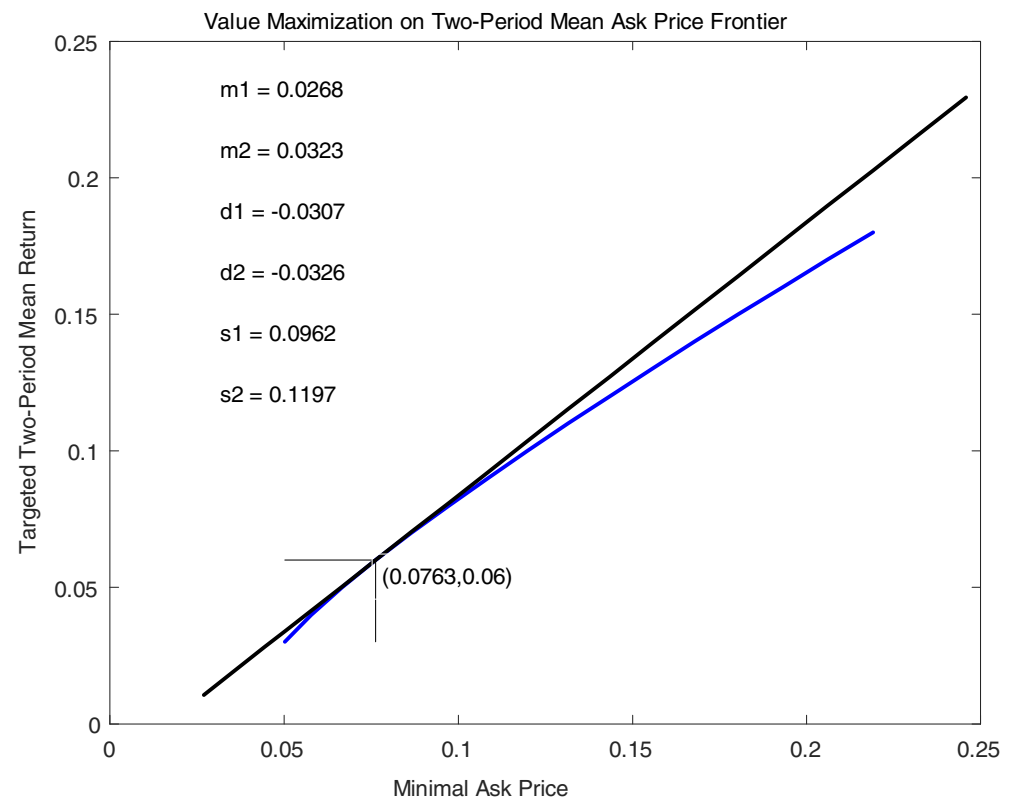

Fig. 8 Mean ask price frontier and value maximizing solution for a higher common component 


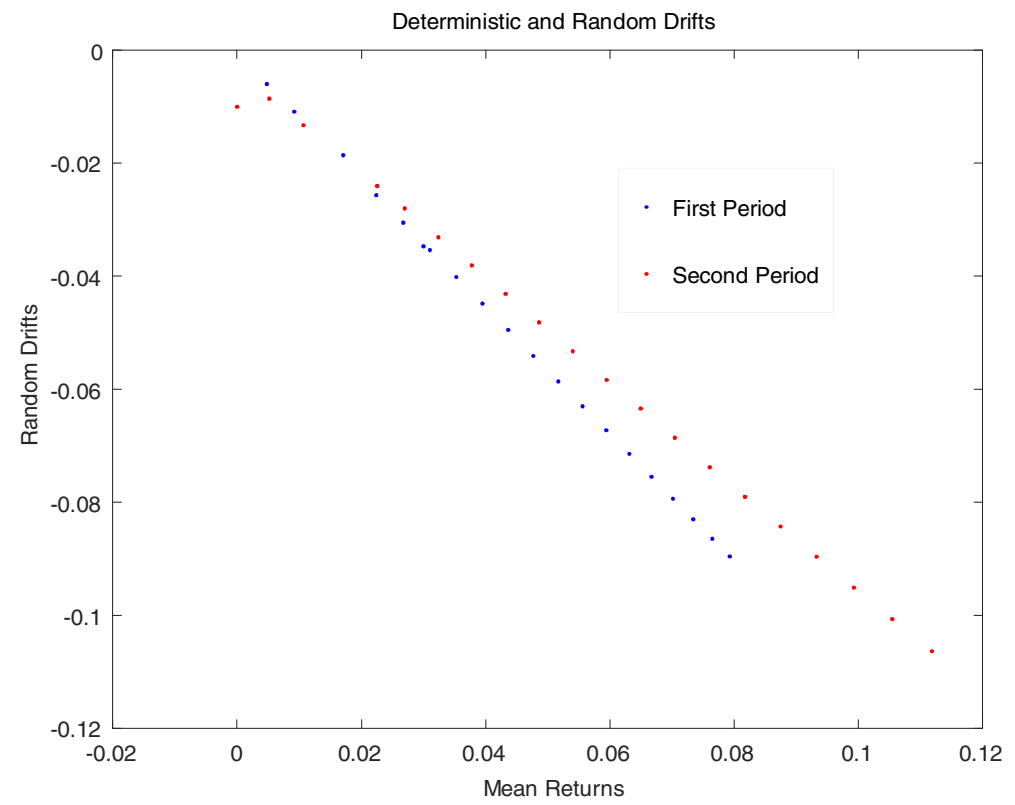

Fig. 9 The random drift as a function of the deterministic drift targeted in each period

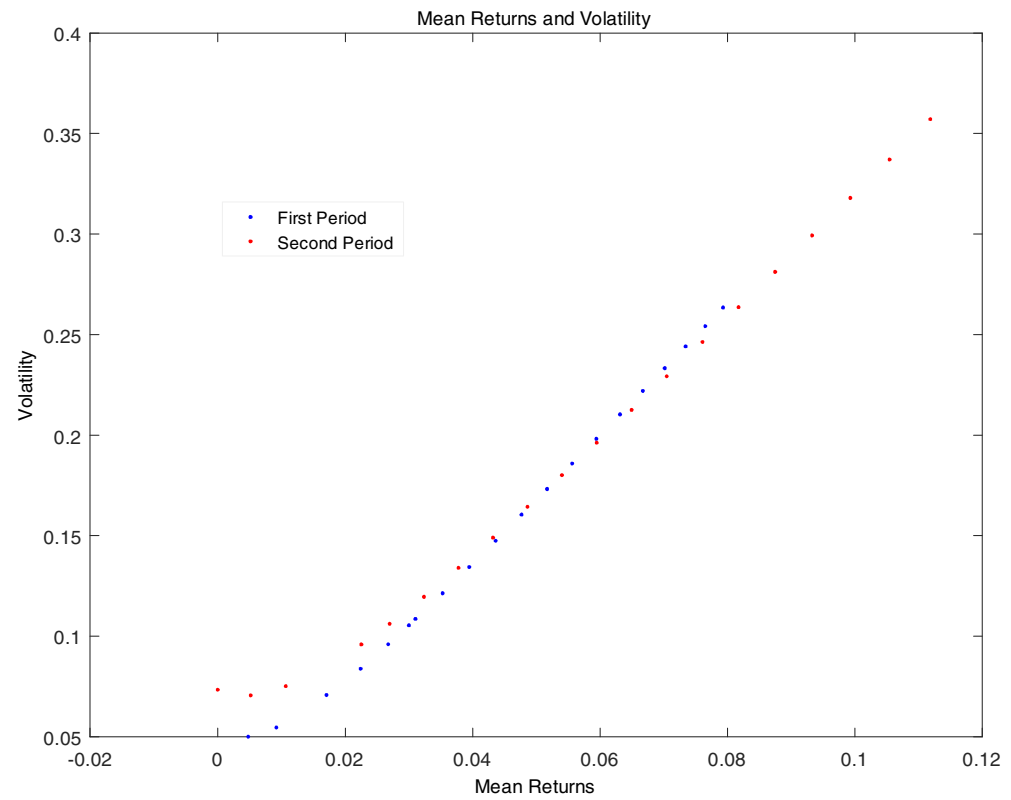

Fig. 10 The volatility targeted as a function of the mean return targeted in each period 


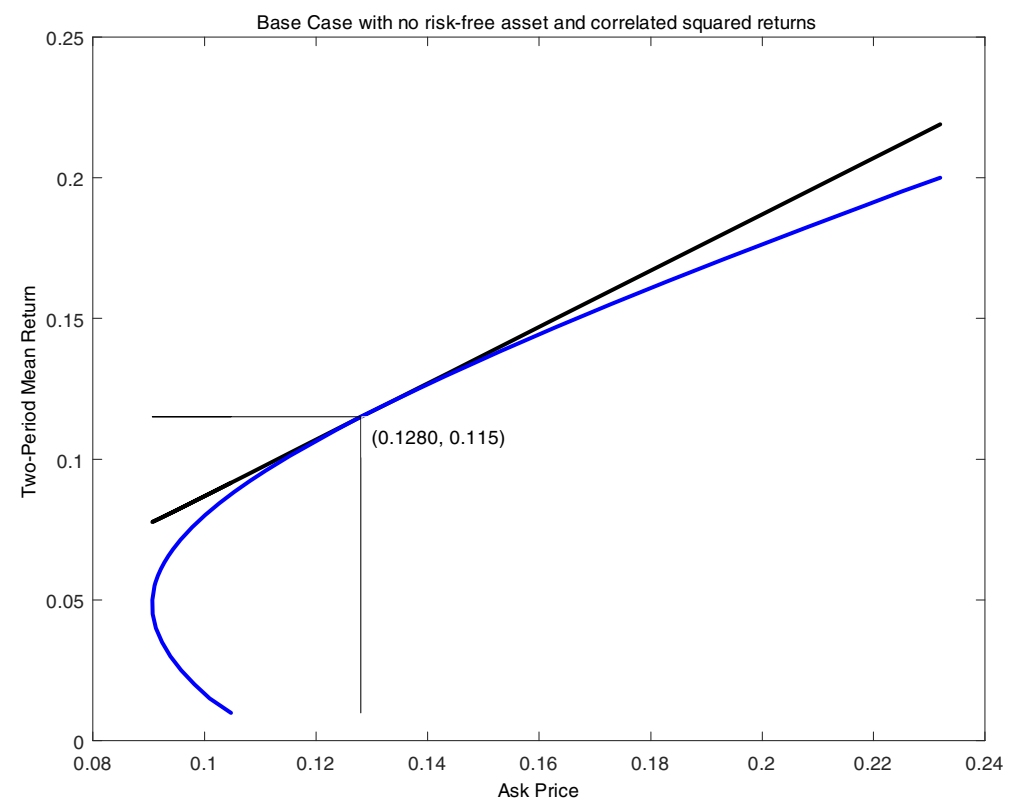

Fig. 11 Mean ask price two-period frontier for correlated squared returns and no random drifts or skewness access

The variance of the two-period return is given by

$$
\begin{aligned}
\operatorname{Variance}\left[\frac{V_{2}}{V_{0}}\right]= & a_{0}^{\prime} \Sigma_{1} a_{0}+a_{1}^{\prime} \Sigma_{2} a_{1} \\
& +a_{0}^{\prime} \Sigma_{1} a_{0} a_{1}^{\prime} \Sigma_{2} a_{1}\left(1+v\left(\alpha_{1} \wedge \alpha_{2}\right)\right) \\
& +2 a_{0}^{\prime} \Sigma_{1} a_{0} \mu_{2}^{\prime} a_{1}+2 a_{1}^{\prime} \Sigma_{2} a_{1} \mu_{1}^{\prime} a_{0}
\end{aligned}
$$

We may observe from Eq. (8) that variances over two periods rise with an increase in the share or variance rate of the component in the time change. From a meanvariance perspective one would expect portfolio adjustments to take place within periods in response to such movements in the joint distribution between periods. Let $\chi=\left(1+v\left(\alpha_{1} \wedge \alpha_{2}\right)\right)$ be the parameter representing henceforth the dependency in the time change. The two-period mean-variance optimization problem in the presence of correlated time changes may then be written, for a risk aversion coefficient of $A$, as maximizing over choices of $a_{0}, a_{1}$, the objective utility $U$,

$U=a_{0}^{\prime} \mu_{1}+a_{1}^{\prime} \mu_{2}+a_{0}^{\prime} \mu_{1} \mu_{2}^{\prime} a_{1}-\frac{A}{2}\left(\begin{array}{c}a_{0}^{\prime} \Sigma_{1} a_{0}\left(1+2 \mu_{2}^{\prime} a_{1}\right)+a_{1}^{\prime} \Sigma_{2} a_{1}\left(1+2 \mu_{1}^{\prime} a_{0}\right) \\ +\chi a_{0}^{\prime} \Sigma_{1} a_{0} a_{1}^{\prime} \Sigma_{2} a_{1}\end{array}\right)$

subject to the constraints

$$
\begin{aligned}
& a_{0}^{\prime} \mathbf{1}=1 \\
& a_{1}^{\prime} \mathbf{1}=1
\end{aligned}
$$


The impact of $\chi$ on portfolio choices in the two periods may be expressed in terms of positions on the two mean-variance efficiency frontiers for the two single periods. For this purpose, we introduce the two frontiers defined by their distinguished spanning portfolios (Skiadas (2009)), the minimum variance portfolios $\zeta_{1}, \zeta_{2}$ for each period and two distinguished efficient portfolios $\eta_{1}, \eta_{2}$ that we shall refer to as the market portfolios for the two periods. These portfolios are given by

$$
\begin{aligned}
& \zeta_{1}=\frac{\Sigma_{1}^{-1} \mathbf{1}}{\mathbf{1}^{\prime} \Sigma_{1}^{-1} \mathbf{1}} \\
& \zeta_{2}=\frac{\Sigma_{2}^{-1} \mathbf{1}}{\mathbf{1}^{\prime} \Sigma_{2}^{-1} \mathbf{1}} \\
& \eta_{1}=\frac{\Sigma_{1}^{-1} \mu_{1}}{\mathbf{1}^{\prime} \Sigma_{1}^{-1} \mu_{1}} \\
& \eta_{2}=\frac{\Sigma_{2}^{-1} \mu_{2}}{\mathbf{1}^{\prime} \Sigma_{2}^{-1} \mu_{2}} .
\end{aligned}
$$

In addition, we introduce the variances $\sigma_{1}^{2}, \sigma_{2}^{2}$ and mean returns $w_{1}, w_{2}$ on the minimum variance portfolios as

$$
\begin{aligned}
\sigma_{1}^{2} & =\frac{1}{\mathbf{1}^{\prime} \Sigma_{1}^{-1} \mathbf{1}} \\
\sigma_{2}^{2} & =\frac{1}{\mathbf{1}^{\prime} \Sigma_{2}^{-1} \mathbf{1}} \\
w_{1} & =\frac{\mathbf{1}^{\prime} \Sigma_{1}^{-1} \mu_{1}}{\mathbf{1}^{\prime} \Sigma_{1}^{-1} \mathbf{1}} \\
w_{2} & =\frac{\mathbf{1}^{\prime} \Sigma_{2}^{-1} \mu_{2}}{\mathbf{1}^{\prime} \Sigma_{2}^{-1} \mathbf{1}} .
\end{aligned}
$$

The mean returns $\rho_{1}, \rho_{2}$ on the market portfolios are given by

$$
\begin{aligned}
& \rho_{1}=\frac{\mu_{1}^{\prime} \Sigma_{1}^{-1} \mu_{1}}{\mathbf{1}^{\prime} \Sigma_{1}^{-1} \mu_{1}} \\
& \rho_{2}=\frac{\mu_{2}^{\prime} \Sigma_{2}^{-1} \mu_{2}}{\mathbf{1}^{\prime} \Sigma_{2}^{-1} \mu_{2}} .
\end{aligned}
$$

Let $m_{1}, v_{1}$ and $m_{2}, v_{2}$ be the mean and variance of two mean-variance efficient portfolios for the two periods. We recall and also review in the appendix the relations between $m, v$ for mean-variance efficient portfolios given by 


$$
\begin{aligned}
& \frac{\left(m_{1}-w_{1}\right)^{2}}{v_{1}-\sigma_{1}^{2}}=\frac{w_{1}}{\sigma_{1}^{2}}\left(\rho_{1}-w_{1}\right) \\
& \frac{\left(m_{2}-w_{2}\right)^{2}}{v_{2}-\sigma_{2}^{2}}=\frac{w_{2}}{\sigma_{2}^{2}}\left(\rho_{2}-w_{2}\right) .
\end{aligned}
$$

Proposition 5 The solution to the two-period mean-variance optimization problem of maximizing $U$ as given by Eq. (9) subject to the constraints (10) and (11) is given by two mean-variance efficient portfolios in the two periods satisfying Eqs. (12) and (13), where the two variances are given by solutions to

$$
\begin{aligned}
v_{1}= & \sigma_{1}^{2}+\frac{w_{1}\left(\rho_{1}-w_{1}\right)}{\sigma_{1}^{2}} \times \\
& \left(\frac{1+w_{2}+\sqrt{\frac{w_{2}}{\sigma_{2}^{2}}\left(\rho_{2}-w_{2}\right)\left(v_{2}-\sigma_{2}^{2}\right)}-A v_{2}}{A\left(1+2\left(w_{2}+\sqrt{\frac{w_{2}}{\sigma_{2}^{2}}\left(\rho_{2}-w_{2}\right)\left(v_{2}-\sigma_{2}^{2}\right)}\right)+\chi v_{2}\right)}\right)^{2} \\
v_{2}= & \sigma_{2}^{2}+\frac{w_{2}\left(\rho_{2}-w_{2}\right)}{\sigma_{2}^{2}} \times \\
& \left(\frac{1+w_{1}+\sqrt{\frac{w_{1}}{\sigma_{1}^{2}}\left(\rho_{1}-w_{1}\right)\left(v_{1}-\sigma_{1}^{2}\right)}-A v_{1}}{A\left(1+2\left(w_{1}+\sqrt{\frac{w_{1}}{\sigma_{1}^{2}}\left(\rho_{1}-w_{1}\right)\left(v_{1}-\sigma_{1}^{2}\right)}\right)+\chi v_{1}\right)}\right)^{2} .
\end{aligned}
$$

The Eqs. (14) and (15) may be solved simultaneously for the volatilities of the two periods with portfolio selections then on the appropriate frontiers. Consider, as an example, a stable frontier across the two periods with $w_{1}=w_{2}=.02, \sigma_{1}=\sigma_{2}=$ $.05, \rho_{1}=\rho_{2}=.07$.

For fixed levels of dependency $\chi$, we let risk aversion range from 1 to 5 . We graph in Fig. 12 the frontier volatility as a function of risk aversion for two levels of dependence in variance as given by $\chi$.

Additionally, we report the common single-period volatility as a solution to a oneperiod problem held in each period and the common two-period volatility (given a stable frontier) held in each period for different values of $\chi$ and comparable risk aversions.

$\begin{array}{llll}\text { A } & \text { single period } & \chi=1.25 & \chi=1.75 \\ 1 & 0.6344 & 0.5073 & 0.4818 \\ 3 & 0.2167 & 0.2057 & 0.2027 \\ 5 & 0.1360 & 0.1330 & 0.1321\end{array}$

We also present in Fig. 13 a graph of the common single-period volatility and the two-period volatility for different risk aversions and two levels of $\chi$. 


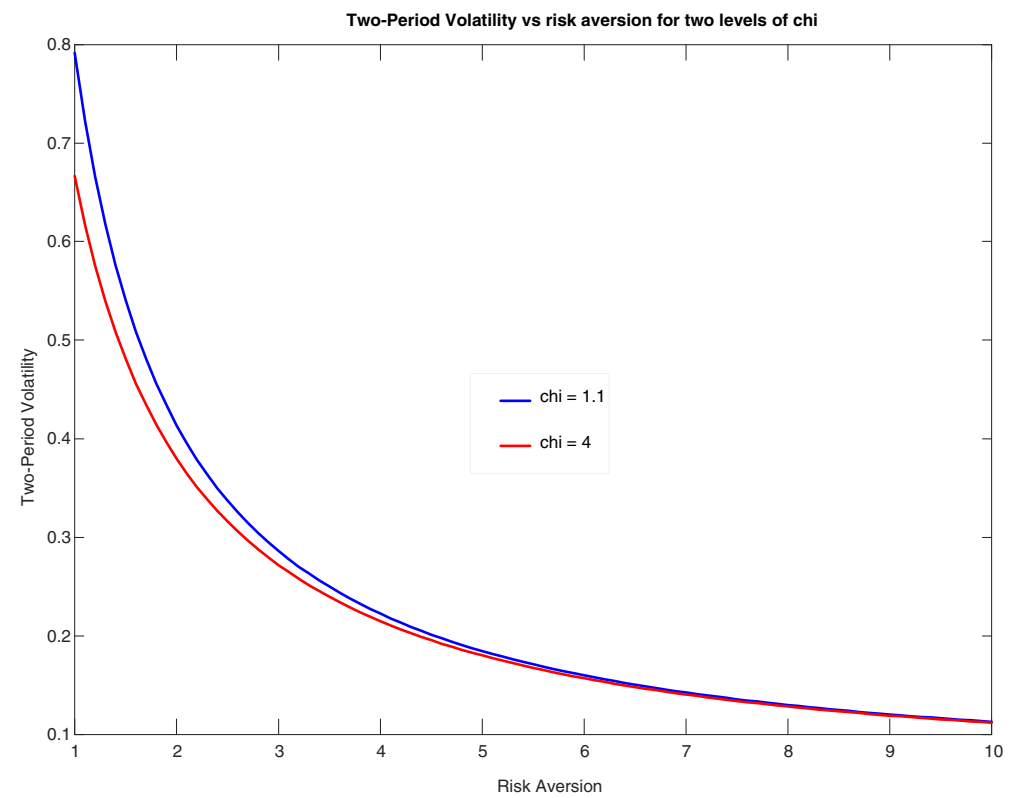

Fig. 12 Frontier volatility as a function of risk aversion for two levels of covariance between random variances for the two periods

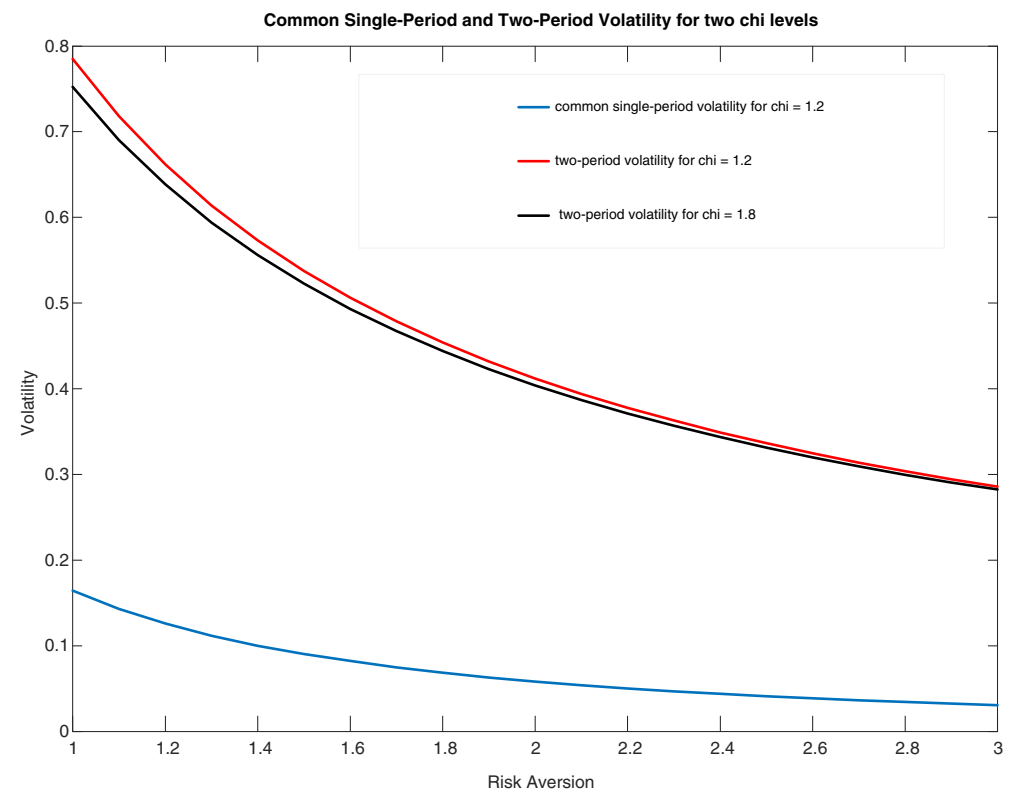

Fig. 13 Single-period and two-period volatilities held by different risk aversions for two levels of $\chi$ 
We observe a reduction in the single-period volatility exposures taken up in the context of correlation in squared returns across periods.

Apart from the solution of the utility maximization problem for the two-period mean-variance problem, one may take up the direct construction of the two-period mean-variance frontier. Here we wish to minimize the two-period variance $V$ subject to attaining a given two-period mean return $m$. Hence, we wish to minimize over choices $a_{0}, a_{1}$

$$
V=a_{0}^{\prime} \Sigma_{1} a_{0}+a_{1}^{\prime} \Sigma_{2} a_{1}+\chi a_{0}^{\prime} \Sigma_{1} a_{0} a_{1}^{\prime} \Sigma_{2} a_{1}+2 a_{0}^{\prime} \Sigma_{1} a_{0} \mu_{2}^{\prime} a_{1}+2 a_{1}^{\prime} \Sigma_{2} a_{1} \mu_{1}^{\prime} a_{0}
$$

subject to

$$
\begin{aligned}
a_{0}^{\prime} \mathbf{1} & =1 \\
a_{1}^{\prime} \mathbf{1} & =1 \\
a_{0}^{\prime} \mu_{1}+a_{1}^{\prime} \mu_{2}+a_{0}^{\prime} \mu_{1} \mu_{2}^{\prime} a_{1} & =m .
\end{aligned}
$$

Proposition 6 The solution of the two-period mean-variance frontier lies on the two single-period efficiency frontiers.

Recognizing that the solution lies on the two single-period frontiers, we may rewrite the problem as one of finding single-period variances $v_{1}, v_{2}$ to minimize

$$
V=v_{1}+v_{2}+\chi v_{1} v_{2}+2 v_{1} m_{2}+2 v_{2} m_{1}
$$

subject to

$$
m_{1}+m_{2}+m_{1} m_{2}=m
$$

where

$$
\begin{aligned}
& m_{1}=w_{1}+\sqrt{\frac{w_{1}}{\sigma_{1}^{2}}\left(\rho_{1}-w_{1}\right)\left(v_{1}-\sigma_{1}^{2}\right)} \\
& m_{2}=w_{2}+\sqrt{\frac{w_{2}}{\sigma_{2}^{2}}\left(\rho_{2}-w_{2}\right)\left(v_{2}-\sigma_{2}^{2}\right)} .
\end{aligned}
$$

Taking the Lagrange multiplier $\lambda$ of the two-period mean constraint as the free variable in place of $m$, we may describe the solution in terms of $\lambda$.

Proposition 7 The system of equations for single-period variances for a twoperiod mean-variance frontier in terms of the two-period mean return Lagrange multiplier is given by 


$$
\begin{aligned}
v_{1}= & \sigma_{1}^{2}+\frac{\lambda^{2}}{4 \frac{w_{1}}{\sigma_{1}^{2}}\left(\rho_{1}-w_{1}\right)} \times \\
& \left(\frac{\left(1+w_{2}+\sqrt{\frac{w_{2}}{\sigma_{2}^{2}}\left(\rho_{2}-w_{2}\right)\left(v_{2}-\sigma_{2}^{2}\right)}\right) \frac{w_{1}}{\sigma_{1}^{2}}\left(\rho_{1}-w_{1}\right)}{1+2\left(w_{2}+\sqrt{\frac{w_{2}}{\sigma_{2}^{2}}\left(\rho_{2}-w_{2}\right)\left(v_{2}-\sigma_{2}^{2}\right)}\right)+\chi v_{2}}\right)^{2} \\
v_{2}= & \sigma_{2}^{2}+\frac{\lambda^{2}}{4 \frac{w_{2}}{\sigma_{2}^{2}}\left(\rho_{2}-w_{2}\right)} \times \\
& \left(\frac{\left(1+w_{1}+\sqrt{\frac{w_{1}}{\sigma_{1}^{2}}\left(\rho_{1}-w_{1}\right)\left(v_{1}-\sigma_{1}^{2}\right)}\right) \frac{w_{2}}{\sigma_{2}^{2}}\left(\rho_{2}-w_{2}\right)}{1+2\left(w_{1}+\sqrt{\frac{w_{1}}{\sigma_{1}^{2}}\left(\rho_{1}-w_{1}\right)\left(v_{1}-\sigma_{1}^{2}\right)}\right)+\chi v_{1}}\right)^{2} .
\end{aligned}
$$

To construct the frontier and the associated solutions we solve Eqs. (16) and (17) for various values of $\lambda$ and then construct $m_{1}, m_{2}$ and $m$ from the equations for the single-period frontier and the constraint equation for $m$. We may then graph against $m$ the values for $v_{1}, v_{2}$. Figure 14 presents a graph of the mean-variance frontiers for the two periods at two different settings for $\chi$. The parameters used were $w_{1}=$ $.02, w_{2}=.01, \sigma_{1}=.05, \sigma_{2}=.03, \rho_{1}=.07, \rho_{2}=.06$.

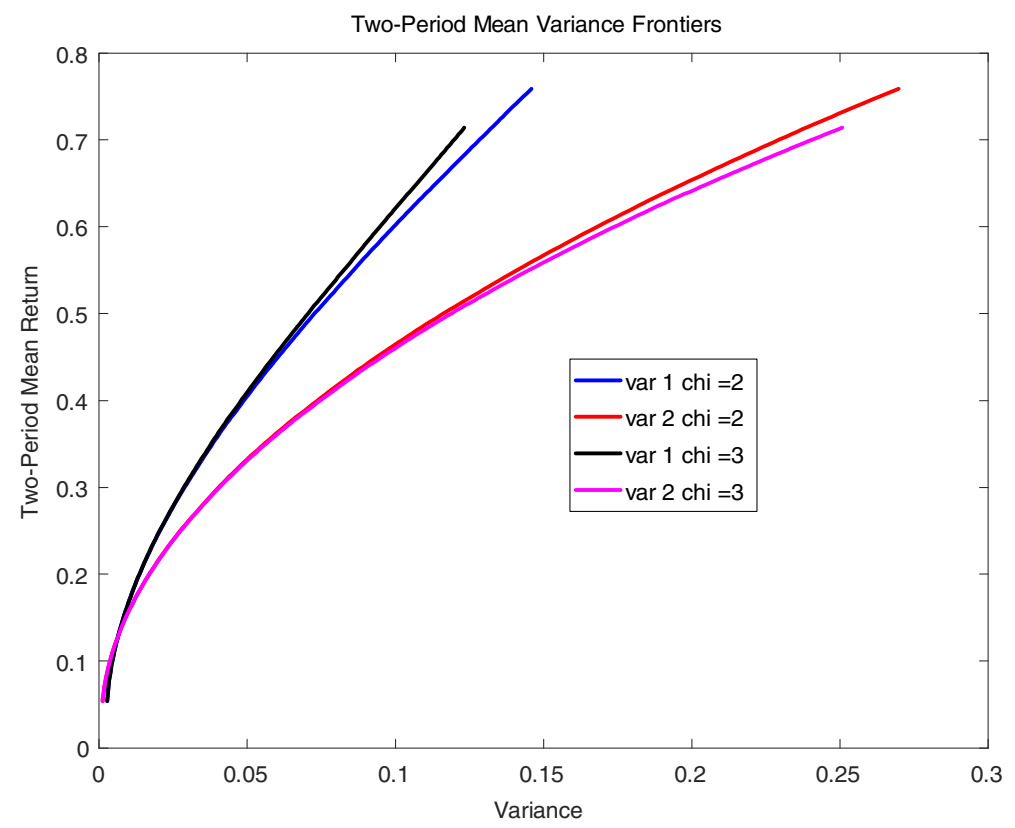

Fig. 14 Mean-variance frontiers for each of the two periods. We present a graph of the two-period mean targeted and the related variances for the two periods at two levels of dependency 
In the presence of risk-free assets in the two periods with risk-free returns of $r_{1}$, $r_{2}$, respectively, the two-period mean return is

$$
E\left[\frac{V_{2}}{V_{0}}\right]=\left(\begin{array}{c}
1+r_{1}+r_{2}+r_{1} r_{2}+ \\
r_{1} a_{1}^{\prime}\left(\mu_{2}-r_{2} \mathbf{1}\right)+r_{2} a_{0}^{\prime}\left(\mu_{1}-r_{1} \mathbf{1}\right)+a_{0}^{\prime}\left(\mu_{1}-r_{1} \mathbf{1}\right)+a_{1}^{\prime}\left(\mu_{2}-r_{2} \mathbf{1}\right) \\
+a_{0}^{\prime}\left(\mu_{1}-r_{1} \mathbf{1}\right)\left(\mu_{2}-r_{2} \mathbf{1}\right)^{\prime} a_{1}
\end{array}\right) .
$$

The two-period variance is given by

$$
\begin{aligned}
\operatorname{Var}\left[\frac{V_{2}}{V_{0}}\right]= & \left(1+r_{2}\right)^{2} a_{0}^{\prime} \Sigma_{1} a_{0}+\left(1+r_{1}\right)^{2} a_{1}^{\prime} \Sigma_{2} a_{1}+\chi a_{0}^{\prime} \Sigma_{1} a_{0} a_{1}^{\prime} \Sigma_{2} a_{1} \\
& +2\left(1+r_{2}\right) a_{0}^{\prime} \Sigma_{1} a_{0} a_{1}^{\prime}\left(\mu_{2}-r_{2} \mathbf{1}\right)+2\left(1+r_{1}\right) a_{1}^{\prime} \Sigma_{2} a_{1} a_{0}^{\prime}\left(\mu_{1}-r_{1} \mathbf{1}\right) \\
& +a_{1}^{\prime} r_{2} \mathbf{1} a_{0}^{\prime} \Sigma_{1} a_{0}\left(a_{1}^{\prime} r_{2} \mathbf{1}-2 a_{1}^{\prime} \mu_{2}\right)+a_{0}^{\prime} r_{1} \mathbf{1} a_{1}^{\prime} \Sigma_{2} a_{1}\left(a_{0}^{\prime} r_{1} \mathbf{1}-2 a_{0}^{\prime} \mu_{1}\right) .
\end{aligned}
$$

The portfolios again lie on the single-period mean-variance frontiers which are now linear and described by

$$
\begin{aligned}
& x_{1}=p_{1} s_{1} \\
& x_{2}=p_{2} s_{2},
\end{aligned}
$$

where $x_{i}=m_{i}-r_{i}$ is the excess return on the portfolio and $s_{i}$ is the standard deviation of the portfolio return. The slope coefficients $p_{i}$ are given by

$$
\begin{aligned}
p_{i} & =\sqrt{\mathbf{1}^{\prime} \Sigma_{i}^{-1}\left(\mu_{i}-r_{i} \mathbf{1}\right) \phi_{i}} \\
\phi_{i} & =\xi_{i}^{\prime}\left(\mu_{i}-r_{i} \mathbf{1}\right) \\
\xi_{i} & =\frac{\Sigma_{i}^{-1}\left(\mu_{i}-r_{i} \mathbf{1}\right)}{\mathbf{1}^{\prime} \Sigma_{i}^{-1}\left(\mu_{i}-r_{i} \mathbf{1}\right)} .
\end{aligned}
$$

\section{Conclusion}

Portfolio theory for two periods is developed in a context allowing for substantial levels of correlation in squared returns while returns are uncorrelated. The returns must then, of necessity, be non-Gaussian making mean-variance analysis less relevant. We develop the two-period conic portfolio problem that leads to a mean ask price frontier. Ask prices are computed using concave distortions and the theory is illustrated and implemented in the context of access to skewness via randomized drifts. The resulting mean-variance frontier is three-dimensional, expressing the minimal variance as a function of the targeted levels for the deterministic and random drift. Optimal portfolios maximize a conservative market value seen as a bid price for the portfolio. On the mean ask price frontier, examples illustrate a trade-off between the deterministic and random drifts and the volatility costs of increasing the deterministic drift.

For completeness, a separate section provides a mean-variance analysis of the two-period portfolio. The result is that in each period one takes positions on the single-period frontier with volatilities satisfying a system of nonlinear equations. Computations illustrate the construction of the two-period frontier. 


\section{Appendix}

Proof of Proposition 1 The Lagrangian is

$$
\mathcal{L}=\frac{1}{2} a^{\prime} \Sigma a-\lambda\left(a^{\prime} \mu-m\right)-\kappa\left(a^{\prime} \theta-d\right)-\pi\left(a^{\prime} \mathbf{1}-1\right) .
$$

The first order condition is

$$
\Sigma a=\lambda \mu+\kappa \theta+\pi \mathbf{1}
$$

with

$$
a=\lambda \Sigma^{-1} \mu+\kappa \Sigma^{-1} \theta+\pi \Sigma^{-1} \mathbf{1} .
$$

In terms of the distinguished portfolios (6), we may write

$$
a=\tilde{\lambda} \eta+\widetilde{\kappa} \delta+\tilde{\pi} \zeta .
$$

The constraints yield

$$
\begin{aligned}
m & =\tilde{\lambda}\left(\rho_{\eta}-\rho_{\zeta}\right)+\widetilde{\kappa}\left(\rho_{\delta}-\rho_{\zeta}\right)+\rho_{\zeta} \\
d & =\tilde{\lambda}\left(y_{\eta}-y_{\zeta}\right)+\widetilde{\kappa}\left(y_{\delta}-y_{\zeta}\right)+y_{\zeta} \\
\tilde{\pi} & =1-\tilde{\lambda}-\tilde{\kappa} .
\end{aligned}
$$

In this case, we have that

$$
v^{*}=\tilde{\lambda}^{2} \sigma_{\eta}^{2}+\widetilde{\kappa}^{2} \sigma_{\delta}^{2}+\tilde{\pi}^{2} \sigma_{\zeta}^{2}+2 \tilde{\lambda} \widetilde{\kappa} \sigma_{\eta \delta}+2 \tilde{\lambda} \tilde{\pi} \sigma_{\eta \zeta}+2 \widetilde{\kappa} \tilde{\pi} \sigma_{\delta \zeta} .
$$

Proof of Proposition 2 With a risk-free asset we have the Lagrangian

$$
\mathcal{L}=\frac{1}{2} a^{\prime} \Sigma a-\lambda\left(a^{\prime}(\mu-r \mathbf{1})-m\right)-\kappa\left(a^{\prime} \theta-d\right) .
$$

The first order condition is

$$
\Sigma a-\lambda(\mu-r \mathbf{1})-\kappa \theta=0 .
$$

Therefore we have that

$$
a=\lambda \Sigma^{-1}(\mu-r \mathbf{1})+\kappa \Sigma^{-1} \theta .
$$

In terms of standardized portfolios we may write

$$
\begin{aligned}
a & =\tilde{\lambda} \xi+\widetilde{\kappa} \delta \\
\xi & =\frac{\Sigma^{-1}(\mu-r \mathbf{1})}{\mathbf{1}^{\prime} \Sigma^{-1}(\mu-r \mathbf{1})} \\
\delta & =\frac{\Sigma^{-1} \theta}{\mathbf{1}^{\prime} \Sigma^{-1} \theta} \\
\tilde{\lambda} & =\mathbf{1}^{\prime} \Sigma^{-1}(\mu-r \mathbf{1}) \lambda \\
\widetilde{\kappa} & =\mathbf{1}^{\prime} \Sigma^{-1} \theta \kappa .
\end{aligned}
$$


Hence $m, d$ can be written in the form

$$
\begin{aligned}
m & =\tilde{\lambda} x_{\xi}+\widetilde{\kappa} x_{\delta} \\
d & =\tilde{\lambda} y_{\xi}+\widetilde{\kappa} y_{\delta},
\end{aligned}
$$

where

$$
\begin{aligned}
& x_{\xi}=\xi^{\prime}(\mu-r \mathbf{1}) ; x_{\delta}=\delta^{\prime}(\mu-r \mathbf{1}) \\
& y_{\xi}=\xi^{\prime} \theta ; y_{\delta}=\delta^{\prime} \theta .
\end{aligned}
$$

Proof of Proposition 3 The variance of $Y_{1}$ is

$$
\begin{aligned}
\sigma_{Y_{1}}^{2} & =E\left[Y_{1}^{2}\right]-\left(E\left[Y_{1}\right]\right)^{2} \\
& =3 \sigma_{1}^{4}-\sigma_{1}^{4} \\
& =2 \sigma_{1}^{4} .
\end{aligned}
$$

The variance of $Y_{2}$ is similarly

$$
\begin{aligned}
\sigma_{Y_{2}}^{2} & =E\left[Y_{2}^{2}\right]-\left(E\left[Y_{2}\right]\right)^{2} \\
& =E\left[\left(\omega+\beta \sigma_{1}^{2}+\alpha \sigma_{1}^{2} Z_{1}^{2}\right)^{2} Z_{2}^{4}\right]-\left(\omega+(\alpha+\beta) \sigma_{1}^{2}\right)^{2} .
\end{aligned}
$$

We expand the first term to get

$$
\left(\left(\omega+\beta \sigma_{1}^{2}\right)^{2}+2 \alpha \sigma_{1}^{2}\left(\omega+\beta \sigma_{1}^{2}\right) Z_{1}^{2}+\alpha^{2} \sigma_{1}^{4} Z_{1}^{4}\right) Z_{2}^{4}
$$

Taking expectations we get

$$
3\left(\omega+\beta \sigma_{1}^{2}\right)^{2}+6 \alpha \sigma_{1}^{2}\left(\omega+\beta \sigma_{1}^{2}\right)+9 \alpha^{2} \sigma_{1}^{4} .
$$

We have to subtract

$$
\begin{aligned}
& \left(\omega+\beta \sigma_{1}^{2}+\alpha \sigma_{1}^{2}\right)^{2} \\
= & \left(\omega+\beta \sigma_{1}^{2}\right)^{2}+2\left(\omega+\beta \sigma_{1}^{2}\right) \alpha \sigma_{1}^{2}+\alpha^{2} \sigma_{1}^{4} .
\end{aligned}
$$

Hence

$$
\begin{aligned}
\sigma_{Y_{2}}^{2} & =2\left(\omega+\beta \sigma_{1}^{2}\right)^{2}+4\left(\omega+\beta \sigma_{1}^{2}\right) \alpha \sigma_{1}^{2}+8 \alpha^{2} \sigma_{1}^{4} \\
& =2 \omega^{2}+4 \omega \beta \sigma_{1}^{2}+2 \beta^{2} \sigma_{1}^{4}+4 \omega \alpha \sigma_{1}^{2}+4 \alpha \beta \sigma_{1}^{4}+8 \alpha^{2} \sigma_{1}^{4} \\
& =\omega\left(2 \omega+4(\alpha+\beta) \sigma_{1}^{2}\right)+2 \beta^{2} \sigma_{1}^{4}+4 \alpha \sigma_{1}^{4}(2 \alpha+\beta) .
\end{aligned}
$$

The denominator for the correlation is then given by

$$
\sqrt{2 \sigma_{1}^{4}\left(\omega\left(2 \omega+4(\alpha+\beta) \sigma_{1}^{2}\right)+2 \beta^{2} \sigma_{1}^{4}+4 \alpha \sigma_{1}^{4}(2 \alpha+\beta)\right)} .
$$


Consider now the covariance computed as the expectation of the product $Y_{1} Y_{2}$ less the product of expectations. The product of expectations is given by

$$
\begin{aligned}
& \sigma_{1}^{2}\left(\omega+(\alpha+\beta) \sigma_{1}^{2}\right) \\
= & \omega \sigma_{1}^{2}+(\alpha+\beta) \sigma_{1}^{4} .
\end{aligned}
$$

For the expectation of the product we have

$$
\begin{aligned}
Y_{1} Y_{2} & =\sigma_{1}^{2} Z_{1}^{2}\left(\omega+\beta \sigma_{1}^{2}+\alpha \sigma_{1}^{2} Z_{1}^{2}\right) Z_{2}^{2} \\
& =\omega \sigma_{1}^{2} Z_{1}^{2} Z_{2}^{2}+\beta \sigma_{1}^{4} Z_{1}^{2} Z_{2}^{2}+\alpha \sigma_{1}^{4} Z_{1}^{4} Z_{2}^{2} .
\end{aligned}
$$

Taking expectations we have

$$
\begin{aligned}
& \omega \sigma_{1}^{2}+\beta \sigma_{1}^{4}+3 \alpha \sigma_{1}^{4} \\
= & \omega \sigma_{1}^{2}+(\alpha+\beta) \sigma_{1}^{4}+2 \alpha \sigma_{1}^{4} .
\end{aligned}
$$

The covariance is then

$$
2 \alpha \sigma_{1}^{4}
$$

and the correlation is

$$
\begin{aligned}
\rho & =\frac{2 \alpha \sigma_{1}^{4}}{\sqrt{2 \sigma_{1}^{4}\left(\omega\left(2 \omega+4(\alpha+\beta) \sigma_{1}^{2}\right)+2 \beta^{2} \sigma_{1}^{4}+4 \alpha \sigma_{1}^{4}(2 \alpha+\beta)\right)}} \\
& =\frac{\alpha}{\sqrt{\frac{\omega^{2}}{\sigma_{1}^{4}}+\frac{2(\alpha+\beta) \omega}{\sigma_{1}^{2}}+\beta^{2}+2 \alpha(2 \alpha+\beta)}} \\
& \leq \frac{\alpha}{\sqrt{\beta^{2}+2 \alpha(2 \alpha+\beta)}} \\
& =\frac{1}{\sqrt{\frac{\beta^{2}}{\alpha^{2}}+4+2 \beta / \alpha}} .
\end{aligned}
$$

Proof of Proposition 4 For the two-period expectation we have

$$
\begin{aligned}
E\left[V_{2}\right] & =V_{0}\left(1+a_{0}^{\prime} \mu_{1}\right)+V_{0} E\left[\left(1+a_{0}^{\prime} R_{1}\right) a_{1}^{\prime} R_{2}\right] \\
& =V_{0}\left(1+a_{0}^{\prime} \mu_{1}\right)+V_{0} a_{1}^{\prime} \mu_{2}+V_{0} E\left[a_{0}^{\prime} R_{1} a_{1}^{\prime} R_{2}\right] \\
& =V_{0}\left(1+a_{0}^{\prime} \mu_{1}\right)+V_{0} a_{1}^{\prime} \mu_{2}+V_{0} E\left[a_{0}^{\prime}\left(X_{1}\left(T_{1}\right)+\mu_{1}\right)\left(X_{2}\left(T_{2}\right)+\mu_{2}\right)^{\prime} a_{1}\right] \\
& =V_{0}\left(1+a_{0}^{\prime} \mu_{1}+a_{1}^{\prime} \mu_{2}+a_{0}^{\prime} \mu_{1} a_{1}^{\prime} \mu_{2}\right) .
\end{aligned}
$$

For the variance of $V_{2} / V_{0}$ we have four terms for

$$
\frac{V_{2}}{V_{0}}=1+a_{0}^{\prime} R_{1}+a_{1}^{\prime} R_{2}+a_{0}^{\prime} R_{1} R_{2}^{\prime} a_{1} .
$$

We thus have 3 terms of variances and 3 covariances. 
First, we evaluate

$$
\begin{aligned}
\operatorname{Var}\left(a_{0}^{\prime} R_{1}\right) & =\operatorname{Var}\left(a_{0}^{\prime} X_{1}\left(T_{1}\right)\right) \\
& =E\left[T_{1}\right] a_{0}^{\prime} \Sigma_{1} a_{0} .
\end{aligned}
$$

Second, we have

$$
\operatorname{Var}\left(a_{1}^{\prime} R_{2}\right)=E\left[T_{2}\right] a_{1}^{\prime} \Sigma_{2} a_{1}
$$

The third variance is

$$
\begin{aligned}
\operatorname{Var}\left(a_{0}^{\prime} R_{1} R_{2}^{\prime} a_{1}\right) & =E\left[\left(a_{0}^{\prime} X_{1}\left(T_{1}\right)\right)^{2}\left(X_{2}\left(T_{2}\right)^{\prime} a_{1}\right)^{2}\right] \\
& =a_{0}^{\prime} \Sigma_{1} a_{0} a_{1}^{\prime} \Sigma_{2} a_{1} E\left[T_{1} T_{2}\right] \\
& =a_{0}^{\prime} \Sigma_{1} a_{0} a_{1}^{\prime} \Sigma_{2} a_{1}\left(1+v\left(\alpha_{1} \wedge \alpha_{2}\right)\right) .
\end{aligned}
$$

Note on taking for unit time, $E\left[T_{1}\right]=E\left[T_{2}\right]=1$ that

$$
\begin{aligned}
E\left[T_{1} T_{2}\right] & =E\left[\left(G\left(\alpha_{1}\right)+G_{1}\left(1-\alpha_{1}\right)\right)\left(G\left(\alpha_{2}\right)+G_{2}\left(1-\alpha_{2}\right)\right)\right] \\
& =E\left[G\left(\alpha_{1}\right) G\left(\alpha_{2}\right)+\alpha_{1}\left(1-\alpha_{2}\right)+\alpha_{2}\left(1-\alpha_{1}\right)+\left(1-\alpha_{1}\right)\left(1-\alpha_{2}\right)\right] \\
& =\alpha_{1} \alpha_{2}+v\left(\alpha_{1} \wedge \alpha_{2}\right)+\alpha_{1}\left(1-\alpha_{2}\right)+\alpha_{2}\left(1-\alpha_{1}\right)+\left(1-\alpha_{1}\right)\left(1-\alpha_{2}\right) \\
& =1+v\left(\alpha_{1} \wedge \alpha_{2}\right) .
\end{aligned}
$$

The nonzero covariances are those of

$$
a_{0}^{\prime} R_{1}, a_{0}^{\prime} R_{1} R_{2}^{\prime} a_{1},
$$

and also the covariance of

$$
a_{1}^{\prime} R_{2}, a_{0}^{\prime} R_{1} R_{2}^{\prime} a_{1} .
$$

For the first, we have to consider the product of

$$
a_{0}^{\prime} X_{1}\left(T_{1}\right), a_{0}^{\prime} R_{1} R_{2}^{\prime} a_{1}-a_{0}^{\prime} \mu_{1} \mu_{2}^{\prime} a_{1} .
$$

So we have the product of

$$
a_{0}^{\prime} X_{1}\left(T_{1}\right), a_{0}^{\prime}\left(\left(\mu_{1}+X_{1}\left(T_{1}\right)\right)\left(\mu_{2}+X_{2}\left(T_{2}\right)\right)^{\prime} a_{1}-a_{0}^{\prime} \mu_{1} \mu_{2}^{\prime} a_{1}\right.
$$

or

$$
\left(a_{0}^{\prime} X_{1}\left(T_{1}\right)\right)^{2} X_{2}\left(T_{2}\right)^{\prime} a_{1}+\left(a_{0}^{\prime} X_{1}\left(T_{1}\right)\right)^{2} \mu_{2}^{\prime} a_{1}+a_{0}^{\prime} X_{1}\left(T_{1}\right) a_{0}^{\prime} \mu_{1} X_{2}\left(T_{2}\right)^{\prime} a_{1} .
$$

The expectation of this term is using conditional independence of $X_{1}, X_{2}$ given $T_{1}, T_{2}$

$$
a_{0}^{\prime} \Sigma_{1} a_{0} \mu_{2}^{\prime} a_{1}
$$

Similarly, we have

$$
a_{1}^{\prime} \Sigma_{2} a_{1} \mu_{1}^{\prime} a_{0} .
$$

Hence, the variance of $V_{2} / V_{0}$ is given by (8).

Proof of Proposition 5 The first order conditions are for Lagrange multipliers $\lambda_{0}, \lambda_{1}$ for the two constraints

$$
\begin{aligned}
& \mu_{1}+\left(\mu_{2}^{\prime} a_{1}\right) \mu_{1}-A a_{1}^{\prime} \Sigma_{2} a_{1} \mu_{1}-A\left(1+2 \mu_{2}^{\prime} a_{1}\right) \Sigma_{1} a_{0}-A \chi a_{1}^{\prime} \Sigma_{2} a_{1} \Sigma_{1} a_{0}-\lambda_{0} \mathbf{1}=0 \\
& \mu_{2}+\left(\mu_{1}^{\prime} a_{0}\right) \mu_{2}-A a_{0}^{\prime} \Sigma_{1} a_{0} \mu_{2}-A\left(1+2 \mu_{1}^{\prime} a_{0}\right) \Sigma_{2} a_{1}-A \chi a_{0}^{\prime} \Sigma_{1} a_{0} \Sigma_{2} a_{1}-\lambda_{1} \mathbf{1}=0,
\end{aligned}
$$


or

$$
\begin{aligned}
& {\left[1+\left(\mu_{2}^{\prime} a_{1}\right)-A a_{1}^{\prime} \Sigma_{2} a_{1}\right] \mu_{1}-\lambda_{0} \mathbf{1}=A\left(1+2 \mu_{2}^{\prime} a_{1}+\chi a_{1}^{\prime} \Sigma_{2} a_{1}\right) \Sigma_{1} a_{0}} \\
& {\left[1+\left(\mu_{1}^{\prime} a_{0}\right)-A a_{0}^{\prime} \Sigma_{1} a_{0}\right] \mu_{2}-\lambda_{1} \mathbf{1}=A\left(1+2 \mu_{1}^{\prime} a_{0}+\chi a_{0}^{\prime} \Sigma_{1} a_{0}\right) \Sigma_{2} a_{1} .}
\end{aligned}
$$

In particular, premultiplying respectively by $a_{0}^{\prime}, a_{1}^{\prime}$

$$
\begin{aligned}
& {\left[1+\left(\mu_{2}^{\prime} a_{1}\right)-A a_{1}^{\prime} \Sigma_{2} a_{1}\right] a_{0}^{\prime} \mu_{1}-\lambda_{0}=A\left(1+2 \mu_{2}^{\prime} a_{1}+\chi a_{1}^{\prime} \Sigma_{2} a_{1}\right) a_{0}^{\prime} \Sigma_{1} a_{0}} \\
& {\left[1+\left(\mu_{1}^{\prime} a_{0}\right)-A a_{0}^{\prime} \Sigma_{1} a_{0}\right] a_{1}^{\prime} \mu_{2}-\lambda_{1}=A\left(1+2 \mu_{1}^{\prime} a_{0}+\chi a_{0}^{\prime} \Sigma_{1} a_{0}\right) a_{1}^{\prime} \Sigma_{2} a_{1} .}
\end{aligned}
$$

So

$$
\begin{aligned}
& \lambda_{0}=\left[1+\left(\mu_{2}^{\prime} a_{1}\right)-A a_{1}^{\prime} \Sigma_{2} a_{1}\right] a_{0}^{\prime} \mu_{1}-A\left(1+2 \mu_{2}^{\prime} a_{1}+\chi a_{1}^{\prime} \Sigma_{2} a_{1}\right) a_{0}^{\prime} \Sigma_{1} a_{0} \\
& \lambda_{1}=\left[1+\left(\mu_{1}^{\prime} a_{0}\right)-A a_{0}^{\prime} \Sigma_{1} a_{0}\right] a_{1}^{\prime} \mu_{2}-A\left(1+2 \mu_{1}^{\prime} a_{0}+\chi a_{0}^{\prime} \Sigma_{1} a_{0}\right) a_{1}^{\prime} \Sigma_{2} a_{1} .
\end{aligned}
$$

We may rewrite in terms of first- and second-period means $m_{1}, m_{2}$ and variances $v_{1}, v_{2}$ defined as

$$
\begin{aligned}
m_{1} & =a_{0}^{\prime} \mu_{1} ; m_{2}=a_{1}^{\prime} \mu_{2} \\
v_{1} & =a_{0}^{\prime} \Sigma_{1} a_{0} ; \quad v_{2}=a_{1}^{\prime} \Sigma_{2} a_{1},
\end{aligned}
$$

that

$$
\begin{aligned}
& \lambda_{0}=\left(1+m_{2}-A v_{2}\right) m_{1}-A\left(1+2 m_{2}+\chi v_{2}\right) v_{1} \\
& \lambda_{1}=\left(1+m_{1}-A v_{1}\right) m_{2}-A\left(1+2 m_{1}+\chi v_{1}\right) v_{2} .
\end{aligned}
$$

Substituting back for $\lambda_{0}, \lambda_{1}$ into the first order conditions we get

$\left(1+m_{2}-A v_{2}\right) \mu_{1}+\left(A\left(1+2 m_{2}+\chi v_{2}\right) v_{1}-\left(1+m_{2}-A v_{2}\right) m_{1}\right) \mathbf{1}=A\left(1+2 m_{2}+\chi v_{2}\right) \Sigma_{1} a_{0}$ $\left(1+m_{1}-A v_{1}\right) \mu_{2}+\left(A\left(1+2 m_{1}+\chi v_{1}\right) v_{2}-\left(1+m_{1}-A v_{1}\right) m_{2}\right) \mathbf{1}=A\left(1+2 m_{1}+\chi v_{1}\right) \Sigma_{2} a_{1}$,

or

$$
\begin{aligned}
& a_{0}=\frac{1+m_{2}-A v_{2}}{A\left(1+2 m_{2}+\chi v_{2}\right)} \Sigma_{1}^{-1} \mu_{1}+\left(v_{1}-\frac{\left(1+m_{2}-A v_{2}\right) m_{1}}{A\left(1+2 m_{2}+\chi v_{2}\right)}\right) \Sigma_{1}^{-1} \mathbf{1} \\
& a_{1}=\frac{1+m_{1}-A v_{1}}{A\left(1+2 m_{1}+\chi v_{1}\right)} \Sigma_{2}^{-1} \mu_{2}+\left(v_{2}-\frac{\left(1+m_{1}-A v_{1}\right) m_{2}}{A\left(1+2 m_{1}+\chi v_{1}\right)}\right) \Sigma_{2}^{-1} \mathbf{1} .
\end{aligned}
$$

It follows that the positions in each period lie on the mean-variance efficient frontiers for each period.

We may then express $a_{0}, a_{1}$ in terms of standard portfolios as

$$
\begin{aligned}
& a_{0}=\frac{\left(1+m_{2}-A v_{2}\right)}{A\left(1+2 m_{2}+\chi v_{2}\right)} \frac{w_{1}}{\sigma_{1}^{2}} \eta_{1}+\left(\frac{v_{1}}{\sigma_{1}^{2}}-\frac{\left(1+m_{2}-A v_{2}\right)}{A\left(1+2 m_{2}+\chi v_{2}\right)} \frac{m_{1}}{\sigma_{1}^{2}}\right) \zeta_{1} \\
& a_{1}=\frac{\left(1+m_{1}-A v_{1}\right)}{A\left(1+2 m_{1}+\chi v_{1}\right)} \frac{w_{2}}{\sigma_{2}^{2}} \eta_{2}+\left(\frac{v_{2}}{\sigma_{2}^{2}}-\frac{\left(1+m_{1}-A v_{1}\right)}{A\left(1+2 m_{1}+\chi v_{1}\right)} \frac{m_{2}}{\sigma_{2}^{2}}\right) \zeta_{2} .
\end{aligned}
$$


On premultiplication by $\mathbf{1}$ we see that the portfolio weights sum to unity.

$$
\begin{aligned}
& \frac{\left(1+m_{2}-A v_{2}\right)}{A\left(1+2 m_{2}+\chi v_{2}\right)} \frac{w_{1}}{\sigma_{1}^{2}}+\frac{v_{1}}{\sigma_{1}^{2}}-\frac{\left(1+m_{2}-A v_{2}\right)}{A\left(1+2 m_{2}+\chi v_{2}\right)} \frac{m_{1}}{\sigma_{1}^{2}}=1 \\
& \frac{\left(1+m_{1}-A v_{1}\right)}{A\left(1+2 m_{1}+\chi v_{1}\right)} \frac{w_{2}}{\sigma_{2}^{2}}+\frac{v_{2}}{\sigma_{2}^{2}}-\frac{\left(1+m_{1}-A v_{1}\right)}{A\left(1+2 m_{1}+\chi v_{1}\right)} \frac{m_{2}}{\sigma_{2}^{2}}=1 .
\end{aligned}
$$

Hence,

$$
\begin{aligned}
& v_{1}=\sigma_{1}^{2}+\frac{\left(1+m_{2}-A v_{2}\right)}{A\left(1+2 m_{2}+\chi v_{2}\right)}\left(m_{1}-w_{1}\right) \\
& v_{2}=\sigma_{2}^{2}+\frac{\left(1+m_{1}-A v_{1}\right)}{A\left(1+2 m_{1}+\chi v_{1}\right)}\left(m_{2}-w_{2}\right) .
\end{aligned}
$$

Furthermore, deleting period subscripts, we have that the excess return on any one-period mean-variance efficient portfolio satisfies

$$
m-w=p(\rho-w),
$$

where $\rho$ is the mean return on $\eta$, the market portfolio given by

$$
\rho=\frac{\mu^{\prime} \Sigma^{-1} \mu}{\mathbf{1}^{\prime} \Sigma^{-1} \mu},
$$

and the mean-variance efficient portfolio in question is

$$
p \eta+(1-p) \zeta .
$$

For $a_{0}, a_{1}$ the value for $p$ is given in Eq. (18), and hence

$$
\begin{aligned}
& m_{1}-w_{1}=\frac{\left(1+m_{2}-A v_{2}\right)}{A\left(1+2 m_{2}+\chi v_{2}\right)} \frac{w_{1}}{\sigma_{1}^{2}}\left(\rho_{1}-w_{1}\right) \\
& m_{2}-w_{2}=\frac{\left(1+m_{1}-A v_{1}\right)}{A\left(1+2 m_{1}+\chi v_{1}\right)} \frac{w_{2}}{\sigma_{2}^{2}}\left(\rho_{2}-w_{2}\right) .
\end{aligned}
$$

We next observe that for any set of mean-variance efficient portfolios we have that

$$
\begin{aligned}
& \frac{\left(m_{1}-w_{1}\right)^{2}}{\left(v_{1}-\sigma_{1}^{2}\right)}=\frac{w_{1}}{\sigma_{1}^{2}}\left(\rho_{1}-w_{1}\right) \\
& \frac{\left(m_{2}-w_{2}\right)^{2}}{\left(v_{2}-\sigma_{2}^{2}\right)}=\frac{w_{2}}{\sigma_{2}^{2}}\left(\rho_{2}-w_{2}\right) .
\end{aligned}
$$

As an aside we recall, again now deleting time subscripts, that on any efficient frontier portfolio with weights $\omega$, we have

$$
\omega=p \eta+(1-p) \zeta,
$$

hence the mean $m$ of $\omega$ is

$$
m=p \rho+(1-p) w,
$$

or that

$$
m-w=p(\rho-w),
$$

so we may write 


$$
p=\frac{m-w}{\rho-w} .
$$

Also the variance of $\omega$ is

$$
\begin{aligned}
v & =\omega^{\prime} \Sigma \omega \\
& =(p \eta+(1-p) \zeta)^{\prime} \Sigma(p \eta+(1-p) \zeta) \\
& =p^{2} \frac{\sigma^{2} \rho}{w}+(1-p)^{2} \sigma^{2}+2 p(1-p) \sigma^{2},
\end{aligned}
$$

so

$$
\begin{aligned}
v-\sigma^{2} & =p^{2} \frac{\sigma^{2} \rho}{w}-2 p \sigma^{2}+p^{2} \sigma^{2}+2 p \sigma^{2}-2 p^{2} \sigma^{2} \\
& =p^{2} \sigma^{2}\left(\frac{\rho-w}{w}\right) \\
& =\frac{(m-w)^{2}}{(\rho-w)^{2}} \frac{\sigma^{2}(\rho-w)}{w} .
\end{aligned}
$$

Hence

$$
\frac{(m-w)^{2}}{v-\sigma^{2}}=\frac{w}{\sigma^{2}}(\rho-w) .
$$

We then rewrite Eqs. (19) and (20) as

$$
\begin{aligned}
& A\left(1+2 m_{2}+\chi v_{2}\right)\left(m_{1}-w_{1}\right)=\left(1+m_{2}-A v_{2}\right) \frac{w_{1}}{\sigma_{1}^{2}}\left(\rho_{1}-w_{1}\right) \\
& A\left(1+2 m_{1}+\chi v_{1}\right)\left(m_{2}-w_{2}\right)=\left(1+m_{1}-A v_{1}\right) \frac{w_{2}}{\sigma_{2}^{2}}\left(\rho_{2}-w_{2}\right) .
\end{aligned}
$$

We note that

$$
\left(m_{1}-w_{1}\right)=\sqrt{\frac{w_{1}}{\sigma_{1}^{2}}\left(\rho_{1}-w_{1}\right)\left(v_{1}-\sigma_{1}^{2}\right)}
$$

and on substitution for $m_{1}-w_{1}$ Eq. (21) yields

$$
A\left(1+2 m_{2}+\chi v_{2}\right) \sqrt{\frac{w_{1}}{\sigma_{1}^{2}}\left(\rho_{1}-w_{1}\right)\left(v_{1}-\sigma_{1}^{2}\right)}=\left(1+m_{2}-A v_{2}\right) \frac{w_{1}}{\sigma_{1}^{2}}\left(\rho_{1}-w_{1}\right),
$$

or that

$$
\begin{aligned}
& \frac{A\left(1+2 m_{2}+\chi v_{2}\right)}{1+m_{2}-A v_{2}}=\sqrt{\frac{w_{1}}{\sigma_{1}^{2}} \frac{\left(\rho_{1}-w_{1}\right)}{\left(v_{1}-\sigma_{1}^{2}\right)}} \\
& \frac{A\left(1+2 m_{1}+\chi v_{1}\right)}{1+m_{1}-A v_{1}}=\sqrt{\frac{w_{2}}{\sigma_{2}^{2}} \frac{\left(\rho_{2}-w_{2}\right)}{\left(v_{2}-\sigma_{2}^{2}\right)}} .
\end{aligned}
$$


Substituting out for $m_{1}, m_{2}$ in terms of $v_{1}, v_{2}$ from the characterization of the frontiers we get that

$$
\begin{aligned}
& \frac{A\left(1+2\left(w_{2}+\sqrt{\frac{w_{2}}{\sigma_{2}^{2}}\left(\rho_{2}-w_{2}\right)\left(v_{2}-\sigma_{2}^{2}\right)}\right)+\chi v_{2}\right)}{1+w_{2}+\sqrt{\frac{w_{2}}{\sigma_{2}^{2}}\left(\rho_{2}-w_{2}\right)\left(v_{2}-\sigma_{2}^{2}\right)}-A v_{2}}=\sqrt{\frac{w_{1}}{\sigma_{1}^{2}} \frac{\left(\rho_{1}-w_{1}\right)}{\left(v_{1}-\sigma_{1}^{2}\right)}} \\
& \frac{A\left(1+2\left(w_{1}+\sqrt{\frac{w_{1}}{\sigma_{1}^{2}}\left(\rho_{1}-w_{1}\right)\left(v_{1}-\sigma_{1}^{2}\right)}\right)+\chi v_{1}\right)}{1+w_{1}+\sqrt{\frac{w_{1}}{\sigma_{1}^{2}}\left(\rho_{1}-w_{1}\right)\left(v_{1}-\sigma_{1}^{2}\right)}-A v_{1}}=\sqrt{\frac{w_{2}}{\sigma_{2}^{2}} \frac{\left(\rho_{2}-w_{2}\right)}{\left(v_{2}-\sigma_{2}^{2}\right)}} .
\end{aligned}
$$

These are two equations in two unknowns $v_{1}, v_{2}$ that must be solved and then we determine $m_{1}, m_{2}, v_{1}, v_{2}$ and the allocations in the two periods.

We may rewrite as

$$
\frac{1}{\left(v_{1}-\sigma_{1}^{2}\right)}=\frac{\sigma_{1}^{2}}{w_{1}\left(\rho_{1}-w_{1}\right)}\left(\frac{A\left(1+2\left(w_{2}+\sqrt{\frac{w_{2}}{\sigma_{2}^{2}}\left(\rho_{2}-w_{2}\right)\left(v_{2}-\sigma_{2}^{2}\right)}\right)+\chi v_{2}\right)}{1+w_{2}+\sqrt{\frac{w_{2}}{\sigma_{2}^{2}}\left(\rho_{2}-w_{2}\right)\left(v_{2}-\sigma_{2}^{2}\right)}-A v_{2}}\right)^{2},
$$

or equivalently (14) and (15).

Proof of Proposition 6 The Lagrangian is

$$
\begin{aligned}
\mathcal{L}= & \frac{1}{2}\left(a_{0}^{\prime} \Sigma_{1} a_{0}+a_{1}^{\prime} \Sigma_{2} a_{1}+\chi a_{0}^{\prime} \Sigma_{1} a_{0} a_{1}^{\prime} \Sigma_{2} a_{1}+2 a_{0}^{\prime} \Sigma_{1} a_{0} \mu_{2}^{\prime} a_{1}+2 a_{1}^{\prime} \Sigma_{2} a_{1} \mu_{1}^{\prime} a_{0}\right) \\
& -\lambda_{0}\left(a_{0}^{\prime} \mathbf{1}-1\right)-\lambda_{1}\left(a_{1}^{\prime} \mathbf{1}-1\right)-\kappa\left(a_{0}^{\prime} \mu_{1}+a_{1}^{\prime} \mu_{2}+a_{0}^{\prime} \mu_{1} \mu_{2}^{\prime} a_{1}-m\right) .
\end{aligned}
$$

The first order conditions are

$$
\begin{aligned}
& \left(1+2 \mu_{2}^{\prime} a_{1}+\chi a_{1}^{\prime} \Sigma_{2} a_{1}\right) \Sigma_{1} a_{0}-\lambda_{0} \mathbf{1}-\left(\kappa\left(1+a_{1}^{\prime} \mu_{2}\right)-a_{1}^{\prime} \Sigma_{2} a_{1}\right) \mu_{1}=0 \\
& \left(1+2 \mu_{1}^{\prime} a_{0}+\chi a_{0}^{\prime} \Sigma_{1} a_{0}\right) \Sigma_{2} a_{1}-\lambda_{1} \mathbf{1}-\left(\kappa\left(1+a_{0}^{\prime} \mu_{1}\right)-a_{0}^{\prime} \Sigma_{1} a_{0}\right) \mu_{2}=0 .
\end{aligned}
$$

Premultiply by $a_{0}^{\prime}, a_{1}^{\prime}$ to get

$$
\begin{aligned}
& \left(1+2 m_{2}+\chi v_{2}\right) v_{1}-\lambda_{0}-\left(\kappa\left(1+m_{2}\right)-v_{2}\right) m_{1}=0 \\
& \left(1+2 m_{1}+\chi v_{1}\right) v_{2}-\lambda_{1}-\left(\kappa\left(1+m_{1}\right)-v_{1}\right) m_{2}=0 .
\end{aligned}
$$

So we have

$$
\begin{aligned}
& \lambda_{0}=\left(1+2 m_{2}+\chi v_{2}\right) v_{1}-\left(\kappa\left(1+m_{2}\right)-v_{2}\right) m_{1} \\
& \lambda_{1}=\left(1+2 m_{1}+\chi v_{1}\right) v_{2}-\left(\kappa\left(1+m_{1}\right)-v_{1}\right) m_{2},
\end{aligned}
$$

substituting for these we get

$\left(1+2 m_{2}+\chi v_{2}\right) \Sigma_{1} a_{0}+\left(\left(\kappa\left(1+m_{2}\right)-v_{2}\right) m_{1}-\left(1+2 m_{2}+\chi v_{2}\right) v_{1}\right) \mathbf{1}-\left(\kappa\left(1+m_{2}\right)-v_{2}\right) \mu_{1}=0$
$\left(1+2 m_{1}+\chi v_{1}\right) \Sigma_{2} a_{1}+\left(\left(\kappa\left(1+m_{1}\right)-v_{1}\right) m_{2}-\left(1+2 m_{1}+\chi v_{1}\right) v_{2}\right) \mathbf{1}-\left(\kappa\left(1+m_{1}\right)-v_{1}\right) \mu_{2}=0$. 
So

$$
\begin{aligned}
& a_{0}=\frac{\left(1+2 m_{2}+\chi v_{2}\right) v_{1}-\left(\kappa\left(1+m_{2}\right)-v_{2}\right) m_{1}}{\left(1+2 m_{2}+\chi v_{2}\right)} \Sigma_{1}^{-1} \mathbf{1}+\frac{\left(\kappa\left(1+m_{2}\right)-v_{2}\right)}{\left(1+2 m_{2}+\chi v_{2}\right)} \Sigma_{1}^{-1} \mu_{1} \\
& a_{1}=\frac{\left(1+2 m_{1}+\chi v_{1}\right) v_{2}-\left(\kappa\left(1+m_{1}\right)-v_{1}\right) m_{2}}{\left(1+2 m_{1}+\chi v_{1}\right)} \Sigma_{2}^{-1} \mathbf{1}+\frac{\left(\kappa\left(1+m_{1}\right)-v_{1}\right)}{\left(1+2 m_{1}+\chi v_{1}\right)} \Sigma_{2}^{-1} \mu_{2} .
\end{aligned}
$$

In terms of standard portfolios we may write

$$
\begin{aligned}
& a_{0}=\frac{\left(1+2 m_{2}+\chi v_{2}\right) v_{1}-\left(\kappa\left(1+m_{2}\right)-v_{2}\right) m_{1}}{\left(1+2 m_{2}+\chi v_{2}\right) \sigma_{1}^{2}} \zeta_{1}+\frac{\left(\kappa\left(1+m_{2}\right)-v_{2}\right)}{\left(1+2 m_{2}+\chi v_{2}\right)} \frac{w_{1}}{\sigma_{1}^{2}} \eta_{1} \\
& a_{1}=\frac{\left(1+2 m_{1}+\chi v_{1}\right) v_{2}-\left(\kappa\left(1+m_{1}\right)-v_{1}\right) m_{2}}{\left(1+2 m_{1}+\chi v_{1}\right) \sigma_{2}^{2}} \zeta_{2}+\frac{\left(\kappa\left(1+m_{1}\right)-v_{1}\right)}{\left(1+2 m_{1}+\chi v_{1}\right)} \frac{w_{2}}{\sigma_{2}^{2}} \eta_{2} .
\end{aligned}
$$

Proof of Proposition 7 The Lagrangian is now

$$
\left.\begin{array}{c}
v_{1}+v_{2}+\chi v_{1} v_{2}+2 v_{1} m_{2}+2 v_{2} m_{1} \\
\left(w_{1}+\sqrt{\frac{w_{1}}{\sigma_{1}^{2}}\left(\rho_{1}-w_{1}\right)\left(v_{1}-\sigma_{1}^{2}\right)}\right) \\
+\left(w_{2}+\sqrt{\frac{w_{2}}{\sigma_{2}^{2}}\left(\rho_{2}-w_{2}\right)\left(v_{2}-\sigma_{2}^{2}\right)}\right) \\
+\left(w_{1}+\sqrt{\frac{w_{1}}{\sigma_{1}^{2}}\left(\rho_{1}-w_{1}\right)\left(v_{1}-\sigma_{1}^{2}\right)}\right)\left(w_{2}+\sqrt{\frac{w_{2}}{\sigma_{2}^{2}}\left(\rho_{2}-w_{2}\right)\left(v_{2}-\sigma_{2}^{2}\right)}\right) \\
-m
\end{array}\right) .
$$

The first order conditions are

$$
\begin{aligned}
& 1+2 m_{2}+\chi v_{2}=\lambda\left(\frac{\frac{w_{1}}{\sigma_{1}^{2}}\left(\rho_{1}-w_{1}\right)}{2 \sqrt{\frac{w_{1}}{\sigma_{1}^{2}}\left(\rho_{1}-w_{1}\right)\left(v_{1}-\sigma_{1}^{2}\right)}}\left(1+w_{2}+\sqrt{\left.\frac{w_{2}}{\sigma_{2}^{2}}\left(\rho_{2}-w_{2}\right)\left(v_{2}-\sigma_{2}^{2}\right)\right)}\right)\right) \\
& 1+2 m_{1}+\chi v_{1}=\lambda\left(\frac{\frac{w_{2}}{\sigma_{2}^{2}}\left(\rho_{2}-w_{2}\right)}{2 \sqrt{\frac{w_{2}}{\sigma_{2}^{2}}\left(\rho_{2}-w_{2}\right)\left(v_{2}-\sigma_{2}^{2}\right)}}\left(1+w_{1}+\sqrt{\left.\frac{w_{1}}{\sigma_{1}^{2}}\left(\rho_{1}-w_{1}\right)\left(v_{1}-\sigma_{1}^{2}\right)\right)}\right) .\right.
\end{aligned}
$$


Substituting for $m_{1}, m_{2}$ from the efficient frontier we get

$$
\begin{aligned}
& 1+2\left(w_{2}+\sqrt{\frac{w_{2}}{\sigma_{2}^{2}}\left(\rho_{2}-w_{2}\right)\left(v_{2}-\sigma_{2}^{2}\right)}\right)+\chi v_{2} \\
= & \lambda\left(\frac{\frac{w_{1}}{\sigma_{1}^{2}}\left(\rho_{1}-w_{1}\right)}{2 \sqrt{\frac{w_{1}}{\sigma_{1}^{2}}\left(\rho_{1}-w_{1}\right)\left(v_{1}-\sigma_{1}^{2}\right)}}\left(1+w_{2}+\sqrt{\frac{w_{2}}{\sigma_{2}^{2}}\left(\rho_{2}-w_{2}\right)\left(v_{2}-\sigma_{2}^{2}\right)}\right)\right) \\
& 1+2\left(\frac{\left.w_{1}+\sqrt{\frac{w_{1}}{\sigma_{1}^{2}}\left(\rho_{1}-w_{1}\right)\left(v_{1}-\sigma_{1}^{2}\right)}\right)+\chi v_{1}}{\frac{w_{2}}{\sigma_{2}^{2}}\left(\rho_{2}-w_{2}\right)}\left(1+w_{1}+\sqrt{\frac{w_{1}}{\sigma_{1}^{2}}\left(\rho_{1}-w_{1}\right)\left(v_{1}-\sigma_{1}^{2}\right)}\right)\right) .
\end{aligned}
$$

Let us observe that

$$
\frac{1+2\left(w_{2}+\sqrt{\frac{w_{2}}{\sigma_{2}^{2}}\left(\rho_{2}-w_{2}\right)\left(v_{2}-\sigma_{2}^{2}\right)}\right)+\chi v_{2}}{\left(1+w_{2}+\sqrt{\frac{w_{2}}{\sigma_{2}^{2}}\left(\rho_{2}-w_{2}\right)\left(v_{2}-\sigma_{2}^{2}\right)}\right) \frac{w_{1}}{\sigma_{1}^{2}}\left(\rho_{1}-w_{1}\right)}=\frac{\lambda}{2 \sqrt{\frac{w_{1}}{\sigma_{1}^{2}}\left(\rho_{1}-w_{1}\right)\left(v_{1}-\sigma_{1}^{2}\right)}} .
$$

On squaring we have

$$
\left(\frac{1+2\left(w_{2}+\sqrt{\frac{w_{2}}{\sigma_{2}^{2}}\left(\rho_{2}-w_{2}\right)\left(v_{2}-\sigma_{2}^{2}\right)}\right)+\chi v_{2}}{\left(1+w_{2}+\sqrt{\frac{w_{2}}{\sigma_{2}^{2}}\left(\rho_{2}-w_{2}\right)\left(v_{2}-\sigma_{2}^{2}\right)}\right) \frac{w_{1}}{\sigma_{1}^{2}}\left(\rho_{1}-w_{1}\right)}\right)^{2}=\frac{\lambda^{2}}{4 \frac{w_{1}}{\sigma_{1}^{2}}\left(\rho_{1}-w_{1}\right)\left(v_{1}-\sigma_{1}^{2}\right)} .
$$

Taking reciprocals we get

$$
\frac{4 \frac{w_{1}}{\sigma_{1}^{2}}\left(\rho_{1}-w_{1}\right)\left(v_{1}-\sigma_{1}^{2}\right)}{\lambda^{2}}=\left(\frac{\left(1+w_{2}+\sqrt{\frac{w_{2}}{\sigma_{2}^{2}}\left(\rho_{2}-w_{2}\right)\left(v_{2}-\sigma_{2}^{2}\right)}\right) \frac{w_{1}}{\sigma_{1}^{2}}\left(\rho_{1}-w_{1}\right)}{1+2\left(w_{2}+\sqrt{\frac{w_{2}}{\sigma_{2}^{2}}\left(\rho_{2}-w_{2}\right)\left(v_{2}-\sigma_{2}^{2}\right)}\right)+\chi v_{2}}\right)^{2} .
$$

On rearrangment we get the results (16) and (17).

\section{Authors' contributions}

Both authors read and approved the final manuscript.

\section{Competing interests}

The authors declare that they have no competing interests. 


\section{References}

Acharya, VV, Pedersen, LH: Asset pricing with liquidity risk. J. Financ. Econ 77, 375-410 (2005)

Ait-Sahalia, Y, Brandt, MW: Variable selection for portfolio choice. J. Financ 56, 1297-1351 (2001)

Bajeux-Besnainou, I, Portait, R: Dynamic asset allocation in a mean-variance framework. Manag. Sci 44, 79-95 (1998)

Bansal, R, Dahlquist, M, Harvey, CR: Dynamic Trading Strategies and Portfolio Choice. Working Paper, Duke University (2004)

Barndorff-Nielsen, OE, Shephard, N: Econometric analysis of realized volatility and its use in estimating stochastic volatility models. J. R. Stat. Soc. B 64, 253-280 (2002)

Basak, S, Chabakauri, G: Dynamic mean-variance asset allocation. Rev. Financ. Stud 23, 2970-3016 (2010)

Bielecki, T, Jin, H, Pliska, SR, Zhou, XY: Continuous-time mean-variance portfolio selection with bankruptcy prohibition. Math. Financ 15, 213-244 (2005)

Brandt, MW, Goyal, A, Santa-Clara, P, Stroud, JR: A simulation approach to dynamic portfolio choice with an application to learning about predictability. Rev. Financ. Stud 18, 831-873 (2005)

Brandt, MW: Portfolio Choice Problems. In: Ait-Sahalia, Y, Hansen, LP (eds.) Handbook of Financial Econometrics, Chapter 5, pp. 269-336. Elsevier, Amsterdam (2009)

Brandt, MW, Santa-Clara, P: Dynamic portfolio selection by augmenting the asset space. J. Financ 61, 2187-2218 (2006)

Campbell, JY, Viceira, LM: Strategic Asset Allocation: Portfolio Choice for Long Term Investors. Oxford University Press, Oxford (2002)

Cherny, A, Madan, D: New measures for performance evaluation. Rev. Financ. Stud 22, 2571-2606 (2009)

Cochrane, JHL: A mean-variance benchmark for intertemporal portfolio theory. J. Financ 69, 1-49 (2014)

Cvitanic, J, Lazrak, A, Wang, T: Implications of the Sharpe ratio as a performance in multi-period settings. J. Econ. Dyn. Control 32, 1622-1649 (2008)

Cvitanic, J, Zapatero, F: Introduction to the Economics and Mathematics of Financial Markets. MIT Press, Cambridge, MA (2004)

Duffie, D, Richardson, H: Mean-variance hedging in continuous time. Ann. Appl. Probab 1, 1-15 (1991)

Hong, H, Scheinkman, J, Xiong, W: Asset float and speculative bubbles. J. Financ 61, 1073-1117 (2006)

Jagannathan, R, Ma, T: Risk reduction in large portfolios: why imposing the wrong constraints helps. J. Financ 58, 1651-1683 (2003)

Kusuoka, S: On law invariant coherent risk measures. Adv. Math. Econ 3, 83-95 (2001)

Leippold, M, Trojani, F, Vanini, P: Geometric approach to multiperiod mean variance optimization of assets and liabilities. J. Econ. Dyn. Control 28, 1079-1113 (2004)

Lim, AEB, Zhou, XY: Mean-variance portfolio selection with random parameters in a complete market. Math. Oper. Res 27, 101-120 (2002)

MacLean, LC, Zhao, Y, Ziemba, WT: Mean-variance versus expected utility in dynamic investment analysis. Comput. Manag. Sci 8, 3-22 (2011)

Madan, DB: Conic portfolio theory. Int. J. Theor. Appl. Financ (2016). doi:10.1142/SO219024916500199

Madan, DB, Pistorius, M, Stadje, M: On Dynamic Spectral Risk Measures and a Limit Theorem. Working Paper, Imperial College, London (2015)

Markowitz, HM: Portfolio selection. J. Financ 7, 77-91 (1952)

Markowitz, HM: Foundations of portfolio theory. J. Financ 46, 469-477 (1991)

Skiadas, C: Asset Pricing Theory. Princeton University Press, Princeton, NJ (2009)

Strotz, RH: Myopia and inconsistency in dynamic utility maximization. Rev. Econ. Stud 23, 165-180 (1956)

Zhou, XY, Li, D: Continuous-time mean-variance portfolio selection: a stochastic LQ framework. Appl. Math. Optim 42, 19-33 (2000) 